\title{
分子电子学的新进展
}

\author{
许晓娜 ${ }^{a}$ 韩宾 $^{b} \quad$ 于釾 $*, b \quad$ 朱艳英*, \\ ( ${ }^{a}$ 燕山大学物理系 秦皇岛 066004) \\ ( ${ }^{b}$ 天津大学化学系 天津市分子光电科学重点实验室 天津 300072)
}

\begin{abstract}
摘要 分子尺度电子学通过构筑基于微尺度电极和单个分子或者少量分子聚集体的 “电极-分子-电极” 结, 研究跨越 分子的电荷输运性质. 它将分子本征化学特性与器件构筑相结合, 考察分子的理化特性与电荷输运的构效关系, 揭示 微尺度的量子输运动力学原理, 并探索基于分子的功能电子器件. 是一个集化学、物理学与微电子学为一体的交叉学 科. 总结整理了分子电子学近些年在器件制备、输运机理及应用方面部分有代表性的进展.
\end{abstract}

关键词 分子电子学; 器件构筑; 电荷传输机理

\section{New Progress in Molecular Electronics}

\author{
$\mathrm{Xu}, \mathrm{Xiaona}^{a} \quad \mathrm{Han} \mathrm{Bin}^{b} \quad \mathrm{Yu}, \mathrm{Xi}^{*}, b \quad$ Zhu, Yanying ${ }^{*, a}$
}

( ${ }^{a}$ Yanshan University, Qinhuangdao 066004, China)

( ${ }^{b}$ Tianjin Key Laboratory of Molecular Optoelectronic Sciences, Department of Chemistry, Tianjin University, Tianjin 300072, China)

\begin{abstract}
Molecular-scale electronics studies the charge transport properties across molecules by constructing "electrode-molecule-electrode" junctions based on the molecular electrodes and single molecule or small amounts of molecular aggregates. It examines the structure-property relationship between the physical and chemical properties of the molecule and the charge transport by combining the intrinsic chemical properties of molecule with device architecture, reveals the micro-scale quantum transport mechanics principle, and explores molecular-based functional electronic devices. It is a research field that integrates chemistry, physics and microelectronics. In this review, we summarize some of the representative progress of molecular electronics in basic research (device preparation, transport mechanism) and applications in recent years.

Keywords molecular electronics; device construction; charge transport mechanism
\end{abstract}

\section{1 引言}

分子(尺度)电子学器件采用单个有机分子或者少量 有机分子聚集体与微尺度电极来构筑分子结, 测量并解 析分子尺度上的光电过程, 以期实现新型基于分子的微 纳光电器件, 譬如分子开关、分子整流器、分子晶体管 等.

20 世纪 50 年代, Feynman ${ }^{[1]}$ 提出了 “从单个分子甚 至原子开始进行组装” 的自下而上(bottom up)的方法, 以替代传统的自上而下 (top down)微加工技术, 来构筑 微尺度功能单元的设想, 并指出在微观尺度领域大有可 为(“There' s plenty room at the bottom”). 70 年代, Ratner 等 ${ }^{[2]}$ 率先提出了分子整流器的设想, 从理论上论证了单 分子器件实现的可能性. 随着 Langmuir-Blodgett(LB)膜, 自组装 (Self-Assembly) ${ }^{[3]}$ 和扫描探针显微镜 (SPM) 等技 术的发展 ${ }^{[4]}$, 微观尺度制备工艺与表征手段得到了进一 步的提高, 使得构筑微尺度的 “电极-分子-电极” 体系 成为可能. Reed 等 ${ }^{[5]}$ 首次利用机械力控制断裂结的方法
(Mechanical Controlled Break Junction, MCBJ)测量了吸 附于两个金电极之间的对苯基二硫醇分子的电荷输运 行为, 测得了对苯基二硫醇分子的伏安特性曲线, 证明 了有机分子的导电性. Tao 和 Paddon-Row 等 ${ }^{[6]}$ 开发了基 于扫描探针的单分子断裂结测量技术(Scanning Probe Microscopy Break Junction, SPM-BJ), 并发展成为测量 单分子电荷输运性质的通用方法. 与此同时, 在金属电 极上的自组装单层膜(self-assembled monolayer, SAM)基 础上利用诸如液态金属作为顶电极的单分子层器件也 逐步发展起来，成为相对于单分子结的另一大类构筑分 子尺度器件的技术和研究电荷输运性质的系统 ${ }^{[7]}$.

在过去的十多年中, 分子电子学的研究取得了快速 的发展, 人们已经初步探索了分子电子学诸多相关的技 术和概念. 首先, 分子本身就可以被当作一个丰富的电 子系统，因为它具有多样的轨道及能级，从而具备实现 多种电学功能的潜力; 其次, 分子种类繁多, 可用的分 子有上百万种，一旦清楚了影响电子传输特性的因素， 可以根据需要“定制分子”，从而“定制功能”; 最后，分

\footnotetext{
*E-mail: xi.yu@tju.edu.cn; yywlxzyy@163.com

Received January 10, 2019; published March 12, 2019.

Project supported by the National Natural Science Foundation of China (No. 21773169).

项目受国家自然科学基金(No. 21773169)资助.
} 
子有独特的化学/生物识别功能, 具有可以直接应用于 生物及医学传感器的前景.

分子电子学是一个新兴的充满活力的研究领域. 近 年来, 有关分子电子学领域发表的英文综述和专著很 多 ${ }^{[821]}$. 比较有代表性的英文综述主要如 Venkataraman 等 ${ }^{[22]}$ 从物理有机化学原理的角度分析了针定基团, 电 极, 分子桥三种必备组分在结构及种类上对单分子器件 性能的影响. $\mathrm{Li}$ 等 ${ }^{[23]}$ 针对分子电子学中纳米间隙电极的 制备及单分子器件进行了详细的整理和总结. Guo 和 Xiang 等 ${ }^{[24]}$ 发表的综述则对该领域进行了最全面的总结 和介绍. Scheer 和 Cuevas ${ }^{[25]}$ 合著的 《Molecular Electronics: An introduction to theory and experiment》是介绍 分子电子学领域的理论和实验最全面也最为详尽的专 著.

相比于大量的英文综述文献, 中文文献则比较少. 典型的中文文献如 Zhang 等 ${ }^{[4]}$ 主要介绍了几种构筑及测 试单分子结的方法, 包括扫描隧道显微镜(STM), 导电 原子力显微镜 (CP-AFM), 扫描隧道显微镜裂结 (STM-BJ), 机械可控裂结 (MCBJ) 及单壁碳纳米管 (SWNT) 技术等. Li 等 ${ }^{[26]}$ 对金属-分子-金属结器件的制 备方法及其对电荷输运的影响进行了阐述. Tian 等 ${ }^{[27]}$ 对 金属-分子-金属结器件的电子传输机理与表征进行了 总结, 着重介绍了分子电子学常见的表征方法, 如电 流-电压, 电流-时间, 转变电压谱(TVS), 散粒噪声及非 弹性电子隧穿谱(IETS)等.

目前, 分子电子学在国内, 无论是化学领域还是物 理领域, 认知度都比较有限, 从事这一前沿交叉研究方 向的课题组不多. 但近些年来, 国内的研究也正在兴起, 部分课题组的研究已经达到国际领先水平. 因此有必要 对这一领域近年的快速发展做一个简要浅显的介绍, 使 国内的相关科研工作者对分子电子学有一个基本的了 解和把握.

\section{2 分子器件的制备}

\section{1 单分子器件的制备}

单分子器件是分子电子学研究中的主要体系之一. 单分子器件的制备最常用的是基于 SPM-BJ 技术和 MCBJ 技术两种. 两种方法都是利用贵金属(主要是金) 的延展性, 在断裂过程中形成原子尺度的微小电极和微 小间隙, 再结合分子自组装, 制备电极-分子-电极结器 件. 最早是通过 SPM 的位置精密控制系统, 利用针尖与 金基底形成分子结 ${ }^{[24]}$.

SPM 技术包括 AFM 和 STM. 其中 AFM 通过探针 悬臂的偏转结合光路反馈针尖与样品的作用, 再通过压 电元件精确控制针尖的位置和与样品的作用力. STM 利 用的是金或者铂-铱丝在拉断过程中形成的原子级尖锐 的针尖, 在与导电基底临近时产生的隧穿电流, 通过电 流反馈控制针尖位置 ${ }^{[28]}$. STM 自诞生起就成为研究电荷
输运特性的重要工具, 起初主要研究平躺吸附于导电基 底表面的分子的电荷隧穿输运, 可以看作是单分子结的 前体.

Tao 等 ${ }^{[29]}$ 利用 STM-BJ 技术结合分子在金电极上的 自组装, 在 STM 针尖和一个放置于待测分子溶液中的 金电极之间通过不断的拉伸而形成裂结，溶液中的分子 自组装到裂结里, 从而得到单分子结器件. 他们分别测 得了烷基硫醇单分子以及 4,4-联吡啶单分子的电导. 并 通过 2,2-联吡啶分子与 4,4-联吡啶分子的电导的对比以 及形成分子结前后的电导图对比验证了所测电导确为 单分子电导. 为以后的单分子电导的测试提供了参考. 具体做法如图 1 所示.
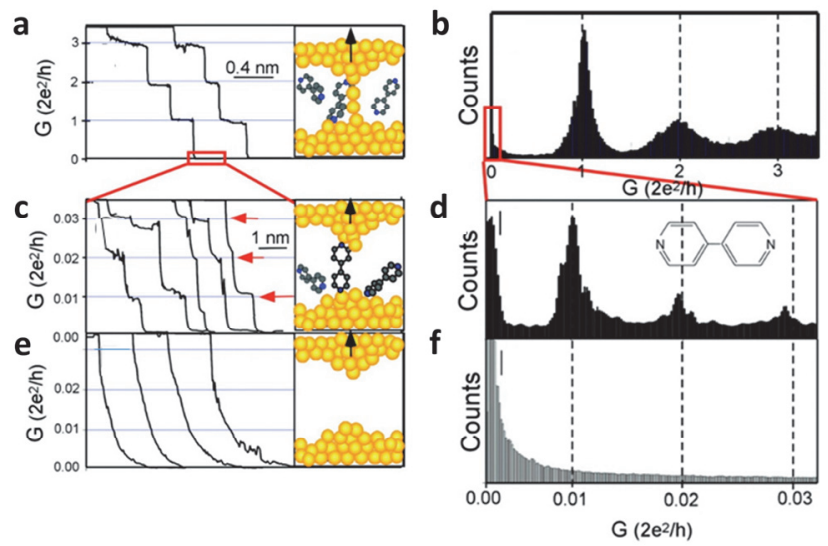

图 1 (a) 随着尖端被拉离基板，在金 STM 尖端和金基板之间形成的 电导以接近 $\mathrm{G}_{0}\left(2 \mathrm{e}^{2} / \mathrm{h}\right)$ 的倍数减小. (b) 与(a)相对应的电导直方图显示 由于电导量子化而在 $1 \mathrm{G}_{0}, 2 \mathrm{G}_{0}$ 和 $3 \mathrm{G}_{0}$ 附近的峰值 ${ }^{[14]}$. (c) 溶液中存在 诸如 4,4'-联吡啶分子, 则出现新的一系列电导台阶. 这些台阶是由于 在尖端和基板电极之间形成了稳定的分子结. (d) (c)对应的电导直方 图, $1 \times, 2 \times$ 和 $3 \times 0.01 \mathrm{G}_{0}$ 附近的峰分别归因于一个, 两个和三个分子. (e 和 f) 在没有分子的情况下在相同的电导范围内没有观察到这样的 台阶或峰 ${ }^{[29]}$

Figure 1 (a) Conductance of a gold contact formed between a gold STM tip and a gold substrate decreases in quantum steps near multiples of $\mathrm{G}_{0}\left(2 \mathrm{e}^{2} / \mathrm{h}\right)$ as the tip is pulled away from the substrate. (b) A corresponding conductance histogram constructed as shown in (a) shows well-defined peaks near $1 \mathrm{G}_{0}, 2 \mathrm{G}_{0}$, and $3 \mathrm{G}_{0}$ due to conductance quantization. (c) A new series of conductance steps appears if molecules such as 4,4-bipyridine are present in the solution. These steps are due to the formation of the stable molecular junction between the tip and the substrate electrodes. (d) (c) Corresponding conductance histogram, peaks near $1 \times$, $2 \times$, and $3 \times 0.01 \mathrm{G}_{0}$ that are ascribed to one, two, and three molecules, respectively. (e and f) In the absence of molecules, no such steps or peaks are observed within the same conductance range $\mathrm{e}^{[29]}$

常规的 STM-BJ 技术是通过针尖与基底间的不断拉 伸来形成分子结并实时监测电导. 由于实验过程中探针 的移动使分子/电极界面变得不稳定, 对电荷输运的研 究产生干扰. Tao 等 ${ }^{[30]}$ 进一步将 STM-BJ 技术进行了改 进. 首先在低偏压下使探针抬起一定高度形成单分子结 后保持静止, 然后在该偏压下测得电流电压曲线. 逐渐 增加偏压重复上述过程直到分子结断裂，如图 2 所示. 该方法能给出在低偏压下的电导随电压清晰的变化关 系，给单分子电导的分析提供更多详细信息，例如可以 
提供单分子的 TVS, 用于揭示分子结构与电荷隧穿之间 细致的关系 ${ }^{[31]}$.
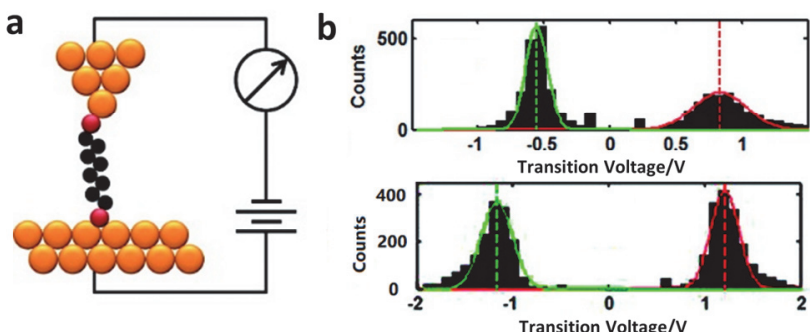

图 2 (a) 烷基二硫醇分子的 STM-BJ 技术示意图. (b) 烷基二硫醇与 联苯二硫醇 TVS 的统计分析 ${ }^{[30]}$

Figure 2 (a) Schematic diagram of STM-BJ technology for alkyl dithiol molecules. (b) Statistical analysis of TVS of alkyldithiols and biphenyldithiols $^{[30]}$

利用 STM-BJ 技术, 近些年人们对微观尺度分子结 的电荷输运进行了细致的研究, 电极与分子的接触及分 子的自身结构对电学特性的影响进行了详细的阐述. Tao 等 ${ }^{[32]}$ 利用 STM-BJ 技术分别研究了颈基、氨基、羧 基三种针定基团对单分子电导的影响, 并发现在三种针 定基团下, 分子结电流-电压曲线在低偏压下是线性的, 增加偏压后由于氨基和羧基与金的相互作用弱而导致 分子结断开. 此外, 氨基和羧基封端的分子对 $\mathrm{pH}$ 值变 化较为敏感, 去质子化以及质子化过程氢离子会影响分 子与金电极之间的耦合强度, 从而影响分子结电导. 该 组之后又报道了关于利用原位电化学的方法制备了单 分子结器件, 提高了器件制备的稳定性及产率 ${ }^{[33]}$. Venkataraman 等 ${ }^{[34]}$ 研究了分子/电极为给-受体连接时分子 结的电学特性, 发现在这种连接下, 单分子器件在较高 偏压下更容易断裂，并且这种断裂有很强的偏压依赖 性, 为研究场击穿提供了一种新方法. Campos 等 ${ }^{[35]}$ 利用 STM-BJ 技术制备了联苯以及联噻吩的单分子结并研究 了分子结构的对称性对于单分子电导的影响. 实验发 现, 当两种分子分别与金电极相连形成分子结时, 由于 对称性的差异观察到联噻吩分子的电导分布较宽, 并且 联噻吩分子相比于对称性较好的联苯分子, 其自由旋转 受器件结构的限制更大, 通过改变噻吩分子的扭转角发 现电导分布对分子的构象有很强的依赖性, 这一结果与 DFT 理论计算结果相一致.

STM-BJ 技术是通过电流反馈针尖位置, 实际上并 不能十分精准地感知分子与电极的接触 ${ }^{[25]}$. 而 AFM 借 助原子力针尖悬臂的变形配合光路的放大效果, 可以感 知针尖与分子的微小作用力. 将 AFM 的硅或者氮化硅 的针尖涂敷一层导电层即可实现 CP-AFM 技术, 可以在 控制分子与电极的接触力的同时进行电学测试. Wold 等 ${ }^{[36]}$ 首次利用 CP-AFM 技术测得了烷基硫醇自组装单 层膜的电学特性. Venkataraman 等 ${ }^{[37]}$ 也利用该技术测得 了联吡啶以及吡啶基-乙烯单分子结在拉伸过程中的电 导变化趋势, 如图 3 所示. 测试结果表明范德华相互作
用对分子结断裂期间局部 $\mathrm{Au}-\mathrm{N}$ 键的伸长与苯环结构 的配位之间的相互作用敏感，在反馈力为 $0.4 \sim 1 \mathrm{nN}$ 时 其对电导有贡献. 该报道对金属/有机界面的范德华相 互作用提供了定量表征.
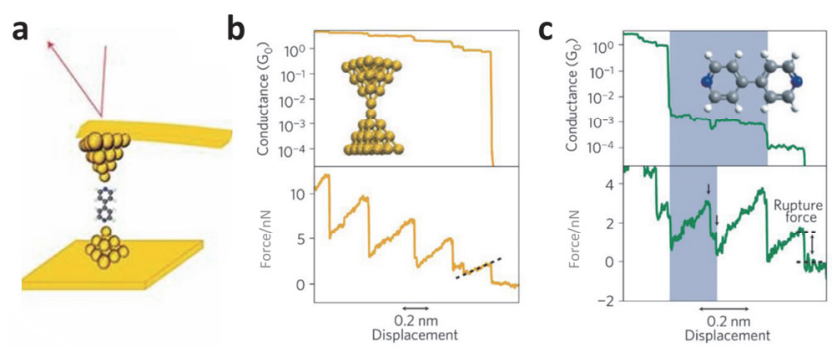

图 3 (a) 利用 CP-AFM 技术在金涂覆的 AFM 尖端和金基底之间形成 的 4,4'-联吡啶结的示意图. (b) 典型的电导-电压曲线以及分子结形成 过程中电导随力的变化. (c) 阴影区域代表高电导分子状态. 下图中 的阴影区域代表高电导状态内的结构重排. 虚线表示分子结完全断 开 ${ }^{[37]}$

Figure 3 (a) Schematic of a 4,4'- bipyridine junction formed between a gold-coated AFM tip and a gold substrate by CP-AFM. (b) Typical current-voltage and conductance-voltage curves and changes in conductance and force during molecular junction formation. (c) The shaded areas represent high-conductance molecular regimes. The shaded areas in the low panel represent structural rearrangements within the highconductance regime. The dashed lines indicate a complete rupture of the molecular junctions ${ }^{[37]}$

大多数裂结技术都无法控制电极与分子的接触 ${ }^{[38]}$, 从而无法避免电导受分子/电极界面的影响. $\mathrm{Xu}$ 等 ${ }^{[39,40]}$ 将传统的 SPM-BJ 技术中探针的连续伸缩分成周期性的 两个过程，先是迅速收回 $0.8 \mathrm{~nm}$, 再停留 $20 \mathrm{~ms}$ 记录电 流和力信号, 如图 4 所示. 该方法能在完全相同的实验 条件下测量多个分子结电导，消除或最小化由于实验条 件的变化造成的测量差异. 该方法可通过改变县臂振幅 来改变针尖与分子的接触力大小, 并且得到输运性质与 调制力之间的关系. 在实验上首次实现了电导的调控, 为分子导线的设计和电学性能可靠的纳米电子结构装 置的构筑提供了指导.
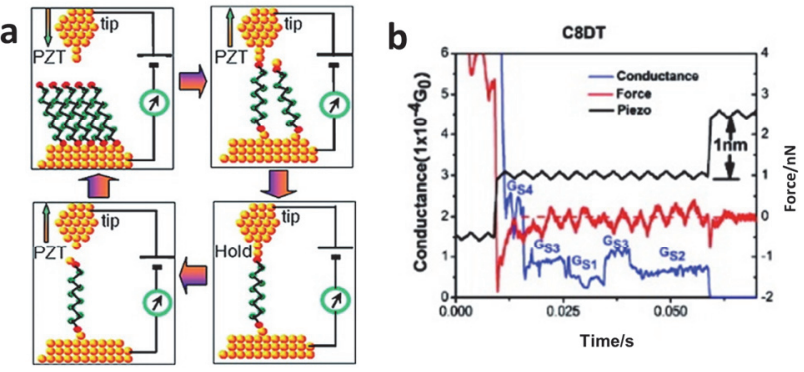

图 4 (a) AFM-BJ 技术示意图. (b) 二硫醇分子的电导与压电元件调 制力以及振幅间的关系图 ${ }^{[39,40]}$

Figure 4 (a) AFM-BJ technology diagram. (b) Diagram of the relationship between the conductance of dithiol molecules and the modulation force and amplitude of piezoelectric elements ${ }^{[39,40]}$

单分子结制备的另一种常用方法 MCBJ 技术首先 由 Moreland 和 Ekin ${ }^{[41]}$ 提出, 之后由 Muller 和 Ruitenbeek 
等 ${ }^{[42]}$ 发展, 直到 1997 年 Reed 等 ${ }^{[5]}$ 首次利用 $\mathrm{MCBJ}$ 技术 测得了苯二硫醇的单分子电导. 其原理如图 5 所示, 在 一个柔性祄底上搭载金属丝，在弯曲梁的反向支撑下通 过压电元件精确控制金属丝断裂形成电极, 利用柔性祄 底的弯曲在电极间形成单分子结. 相对于扫描探针为基 础的断裂结技术, MCBJ 的主要优点是可以通过柔性祄 底和杜杆原理, 配合压电元件在金属电极之间形成连续 可控的间隙, 进而形成机械稳定的单分子结器件. 该装 置中的压电元件相较于 STM 以及 AFM 设备来说尺寸较 小, 便于放置于不同环境下测试单分子器件, 或与其他 测试手段实现集成, 因此成为单分子电子学研究的另一 主流方法. 以此方法为基础结合其他技术可对分子电子 学器件进行更细致的研究.
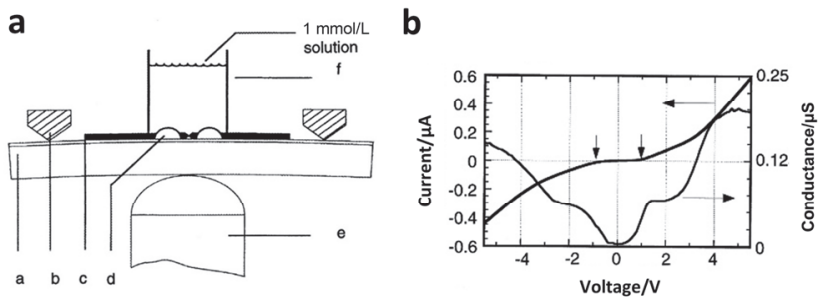

图 5 (a) MCBJ 示意图, 其中 $a, b, c, d, e, f$ 分别为弯曲梁、反向支撑、 缺口金线、胶连处、压电元件以及玻璃管. (b) 典型的苯二硫醇单分子 电流-电压及电导-电压曲线 ${ }^{[5]}$

Figure 5 (a) MCBJ schematic diagram where a, b, c, d, e, f are curved beam, reverse support, notched gold wire, glue joint, piezoelectric element and glass tube. (b) Typical benzenedithiol single molecule current-voltage and conductance-voltage curves $^{[5]}$

将 MCBJ 与光谱技术相结合可以提供原位的精细 的分子结的结构信息, 为电荷输运机理的研究提供有力 支持, 也是 MCBJ 技术近些年来的一个主要进步. 例如 Tian 和 Tao 等 ${ }^{[43]}$ 利用 MCBJ 技术与表面增强拉曼(SERS) 技术结合，原位测试电极间苯二硫醇分子结的拉曼信 号. 通过对比电极不同部位的拉曼信号结合 FDTD 理论 发现电极间隙处拉曼信号最强. 同时通过机械调控电极 间隙发现不同分子的拉曼信号随着间隙的减小而逐渐 增强, 并且电极间的分子拉曼信号有一定的增强. 该方 法实现了对单分子结光学特性的原位检测, 同时也利用 $\mathrm{MCBJ}$ 技术可控的机械稳定性在分子结及电极间得到了 较强的拉曼信号. 使得 MCBJ 及 SERS 技术成为研究分 子电子学器件性质的强有力手段, 为单分子的光学特性 提供了更深刻认识.

此外, 该技术相对于 SPM-BJ 技术, 更容易通过微 加工方法构筑栅极, 从而实现单分子晶体管器件. 如 Mayer 和 Lee 等 ${ }^{[44]}$ 利用 MCBJ 技术在距离苯二硫醇分子 结几纳米处加侧栅极构筑机械稳定的单分子晶体管, 如 图 6 所示. 结合 DFT 理论, 通过栅极调控改变分子结的 电场使分子中的电子结构发生改变进而能级发生位移 来调控分子结电导. 除实现栅极调控外, 通过测试发现 器件的漏电流在 $\mathrm{pA}$ 量级远小于分子的电流范围, 满足 了晶体管的两大重要指标. 为制备机械稳定的单分子晶
体管提供了切实可行的新方法.
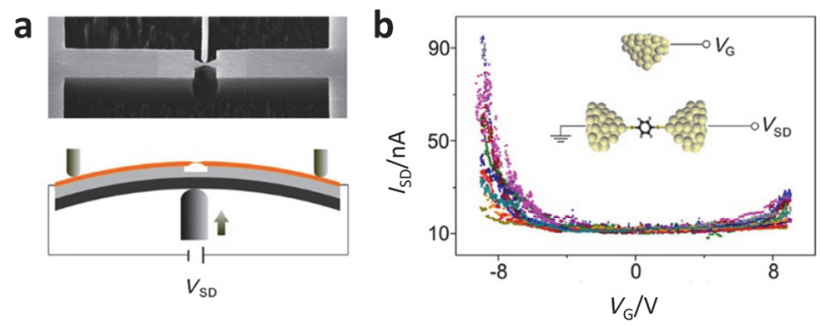

图 6 (a) 对 MCBJ 技术制备的单分子结加栅极的示意图. (b) 苯二硫 醇分子结的电流与栅极电压间的关系图 ${ }^{[44]}$

Figure 6 (a) Schematic diagram of a single molecule junction apply gate formed by MCBJ technology. (b) Diagram of the relationship between the current of 1,4-benzenedithiol molecular knot and the gate volt$\operatorname{age}^{[44]}$

\section{2 单分子层器件的制备}

目前, 单分子器件代表了小型化的一个极限, 而且 由于其相对简单清晰的结构, 理论上处理起来更容易, 因而, 更多地受到物理学家的青睐. 但单分子器件制备 工艺复杂、设备昂贵, 且电导测试需要做大量的统计. 而单分子层器件恰好可弥补这些不足, 它制备工艺简 单, 成本相对较低, 用普通材料和在化学实验室即可实 现.

对于单分子层器件, 分子通常通过自组装与底电极 相连形成单分子膜, 然后在分子层上构建顶电极形成分 子结. 由于极小的分子层厚度, 顶电极的构建以及与分 子层的相互作用就成为分子层器件最关键的技术问题. 顶电极既要与分子层有好的接触, 又要避免破坏分子 层.

早期曾经采用采滴作为顶电极 ${ }^{[45]}$ 与分子层形成紧 密的接触. 但由于液态采有毒且容易与底电极金属形成 采齐从而造成短路 ${ }^{[7]}$ ，其应用受到很大的限制. 而液态 金属铟镓合金 (EGaIn) 由于易被氧化会在表面形成 $\mathrm{Ga}_{2} \mathrm{O}_{3}$ 保护层可以防止金属渗透. 基于此, Whitesides 等 $\left.{ }^{[46} 48\right]$ 率先利用 EGaIn 代替 $\mathrm{Hg}$ 做顶电极, 测得自组装 单分子层的电学特性. 随后, Nijhuis 等 ${ }^{[49]}$ 也利用 EGaIn 作为顶电极制备了自组装的硫醇单分子层器件, 并表征 了器件的稳定性及电学特性, 如图 7 所示. 该技术已经 逐步发展成为一种简便快捷的制备分子层器件的标准 方法并得到广泛应用.

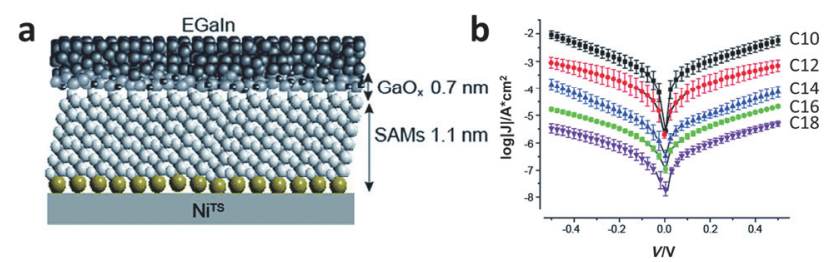

图 7 (a) NiTS-SAM $/ / \mathrm{GaOx} / \mathrm{EGaIn}$ 结的示意图. (b) 分子结的电压与 电流密度对数平均值的关系图 ${ }^{[49]}$

Figure 7 (a) Schematic of the NiTS-SAM//GaO $/$ EGaIn junctions. (b) Plot of the log-average value $J$ - $V$ of junctions with molecular junction ${ }^{[49]}$ 
另一种方法是通过蒸镀金属于单层膜上形成顶电 极. 然而在硫醇分子层上沉积的金或钛会渗透破坏分子 层 ${ }^{[00-54]}$. 为确保分子层结构的完整性, 可通过室温沉积 金属原子的 “软” 沉积技术 ${ }^{[55 ~ 57]}$ 以及在分子层与电极间 加保护层等方法. 例如, Boer 等 ${ }^{[58]}$ 利用光刻等技术在烷 基硫醇自组装分子层与金属顶电极间插入 PEDOT:PSS 保护层, 成功避免了顶电极对分子层的破坏, 大大提高 了分子结的稳定性、重复性及产率. 还有人利用相似的 方法测得了 DNA 双螺旋的单分子层电导, 证明了其与 烷基硫醇分子的电荷传输机制相同 ${ }^{[59]}$.

最近, Lörtscher 等 ${ }^{[60]}$ 将金属纳米粒子自组装在烷基 二硫醇分子层与蒸镀的顶电极之间作为保护层, 实现了 稳定可重复的分子器件. 相较于其他的保护层(石墨烯, 导电高分子), 此方法利用微加工技术制备多种不同孔 径的阵列使纳米粒子在其中与分子层通过化学吸附相 接触. 一方面, 有利于精确控制两端分子与电极的接触 面积, 实现大规模单分子层器件阵列. 另一方面, 化学 吸附可以使金属电极与分子层的接触更紧密. 此方法在 不破坏分子层的同时满足了制备单分子层器件的要求, 也可在低温条件下结合其他测试手段例如非弹性电子 隧穿谱(IETS)对器件进行其他性质的表征. 为将分子化 合物整合到固态器件中提供了新途径, 并且尺寸可缩小 到单分子水平, 如图 8 所示.

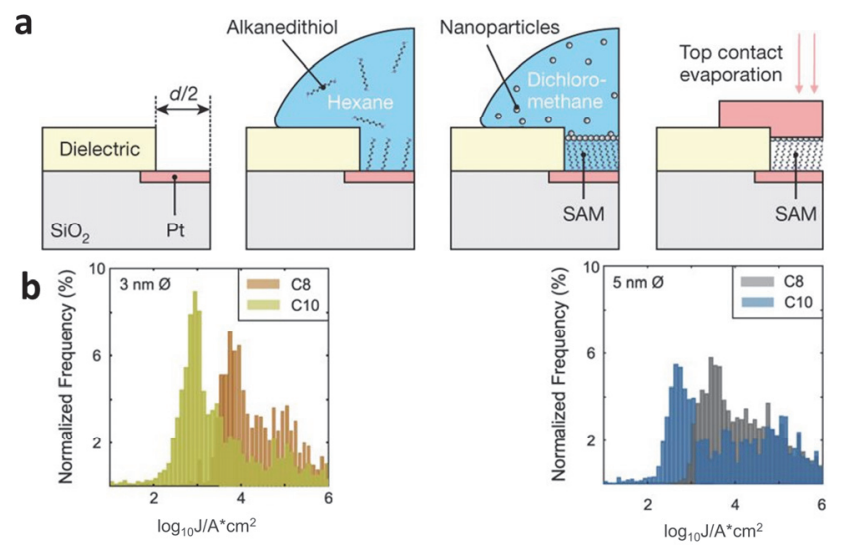

图 8 (a) 金纳米粒子做保护层的器件制备流程图. (b) 不同尺寸的金 纳米粒子对于分子结电学特性的影响 ${ }^{[60]}$

Figure 8 (a) Device preparation process using gold nanoparticle as a protective layer. (b) Effect of different sizes of gold nanoparticles on the electrical properties of molecular junctions ${ }^{[60]}$

除上述方法外，另一个策略是利用悬浮纳米线法制 备单分子层器件. 该技术首先利用微加工的方法制备出 间距在几微米的电极阵列, 然后将分子自组装在金纳米 线上, 再通过介电泳力使金纳米线与底端微电极相连, 在介电泳力的作用下使纳米线与底电极紧密接触, 同时 该力很小不足以破坏分子层. Selzer 等 ${ }^{[6]]}$ 采用自组装悬 浮纳米线的方法制备烷基硫醇单分子层器件并利用 IETS 及 TVS 研究了形成的两个分子结的有效性. 同时 利用纳米线与底电极间产生的表面等离基元研究分子 结电导与入射光功率间的变化关系，如图 9 所示.
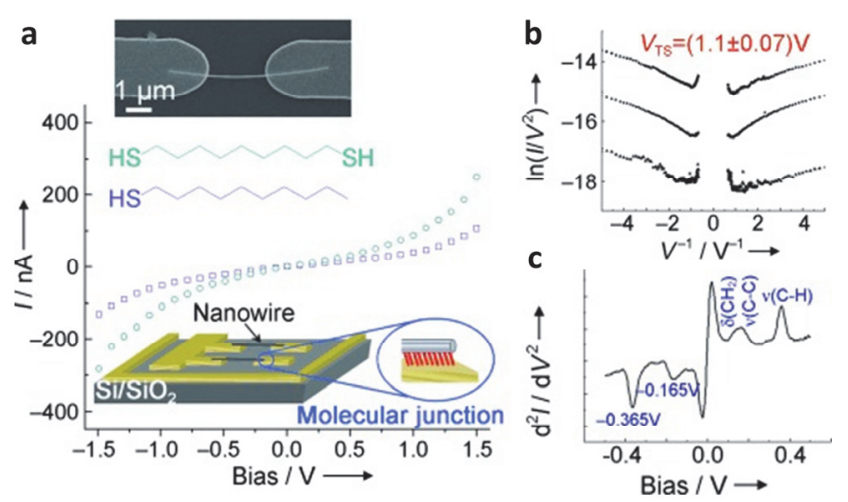

图 9 (a) “悬线” 分子结器件和典型的 $I-V$ 曲线. 插图为悬浮纳米线 的 SEM 图像. (b) 三种不同的 $\mathrm{C} 9$ 硫醇分子结的转变电压谱. (c) 在 $5 \mathrm{~K}$ 下测量的 C9 硫醇分子结的代表性 IETS 谱, 显示烷基链的特征峰(C10 硫醇分子结具有相似的峰 $)^{[61]}$

Figure 9 (a) Representation of a "suspended-wire" molecular junction and typical $I-V$ curves of the molecules used in this study, inset: SEM image of a suspended nanowire. (b) Transition voltage spectroscopy of three different $\mathrm{C} 9$ junctions. (c) Representative IETS measurements of a C9 junction measured at $5 \mathrm{~K}$, showing characteristic peaks of alkyl chains (C10 junctions have similar peaks $)^{[61]}$

\section{3 电荷输运机理及表征}

对于微观尺度分子结中的电荷输运，目前公认的传 输机制有两种, 一种是隧穿机制, 通常指电子经过一步 量子隧穿从一侧电极直接到达另一侧电极. 另一种是跳 跃机制，通常指电子经过一步或多步到达分子，再由分 子到达另一端电极, 如图 10 所示 ${ }^{[2]}$.
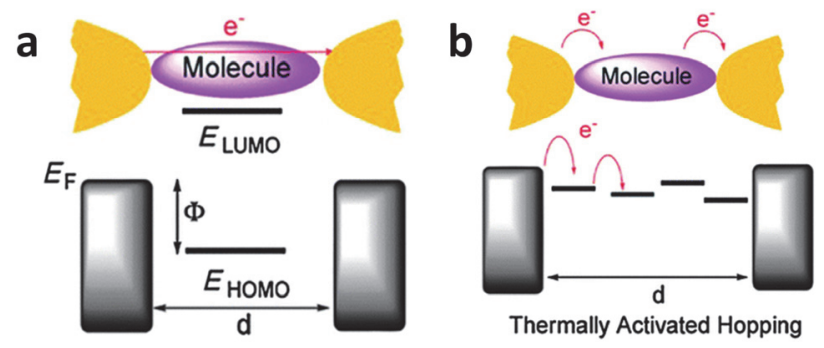

图 10 (a) 隧穿过程示意图. (b) 跳跃过程示意图 ${ }^{[62]}$

Figure 10 (a) The schematic of the tunneling process. (b) Schematic diagram of the hopping process ${ }^{[62]}$

\section{1 隧穿机理}

在简化的隧穿模型中, 分子被简化成一个有一定能 量高度和一定长度的能垒. 分子中的电荷输运是通过矩 形势垒的量子隧穿，可以通过 Simmon's 模型来描述:

$$
J=\frac{e}{4 \pi^{2} \hbar d^{2}}\left\{\begin{array}{l}
\left(\varphi-\frac{e V}{2}\right) \exp \left[-\frac{2(2 m)^{1 / 2}}{\hbar} \alpha\left(\varphi-\frac{e V}{2}\right)^{1 / 2} d\right]- \\
\left(\varphi+\frac{e V}{2}\right) \exp \left[-\frac{2(2 m)^{1 / 2}}{\hbar} \alpha\left(\varphi+\frac{e V}{2}\right)^{1 / 2} d\right]
\end{array}\right\}
$$

其中 $\varphi, d, m$ 和 $V$ 分别代表势垒高度、势垒宽度、电子 质量和施加的偏压, $\alpha$ 是无单位参数, 用于调整隧穿电 
子的有效质量并修改矩形势垒模型. 通过将实验数据和 该公式进行拟合, 可得到有关电荷输运的关键物理量, 例如势垒高度和势垒宽度等. 此外 Simmon's 模型得到 的 $I-V$ 关系可根据电流对偏压的依赖性分为高偏压区和 低偏压区. 在高偏压区(电流一电压非线性依赖) $I-V$ 关系 为:

$I=\left(\frac{e}{4 \pi^{2} \hbar d^{2}}\right)\left(\varphi-\frac{e V}{2}\right) \exp \left[-\frac{2(2 m)^{1 / 2}}{\hbar} \alpha\left(\varphi-\frac{e V}{2}\right)^{1 / 2}\right]$

在低偏压区(电流-电压线性依赖) $I-V$ 关系为:

$I=\left(\frac{(2 m \varphi)^{1 / 2} e^{2} \alpha}{\hbar d^{2}}\right) V \exp \left[-\frac{2(2 m)^{1 / 2}}{\hbar} \alpha(\varphi)^{1 / 2} d\right]$

从上式可以发现, 在低偏压区, 随着势垒宽度(分 子长度)增加, 隧穿电流指数衰减. 这一长度依赖性可 以简化为: $I=I_{0} e^{-\beta d}$, 其中 $\beta$ 为衰减因子, $I_{0}$ 为接触电 流. 可以看出 $\beta \propto \alpha(\varphi)^{1 / 2} d$, 即衰减因子的大小与势垒 高度直接相关, 势垒越高, 衰减因子越大. 这种电导随 分子长度指数衰减的现象可当做判断电荷隧穿输运机 理的一种手段. 对于不同类型的分子结, 隧穿势垒可以 认为是分子的 HOMO 或 LUMO 与电极费米能级间的能 量差. 由于共轭分子比烷基硫醇分子的能量差小, 所以 电导较大, 衰减因子较小.

实验中发现不同结构的同种类型的分子结有相似 的衰减因子, 饱和烷烃为 0.7 和 $0.9 \AA^{-1}$, 共轭分子为 0.2 和 $0.5 \AA^{-1[63]}$. 对于隧穿机制, 由于室温下温度对电极中 电子的费米分布影响很小, 且隧穿机制下电子基本不与 分子发生相互作用. 分子的热运动不会影响电子隧穿, 因此，隧穿机制是没有温度依赖性的.

Reed 等 ${ }^{[64]}$ 最早在实验上基于自组装技术研究不同 长度的烷基硫醇分子结的电荷输运机理, 如图 11 所示, $\mathrm{a} 、 \mathrm{~b}$ 分别给出了实验所得的 $I-V$ 曲线与理论的拟合以及 电流随温度变化的曲线. 从图中可以看出, 实验上分子
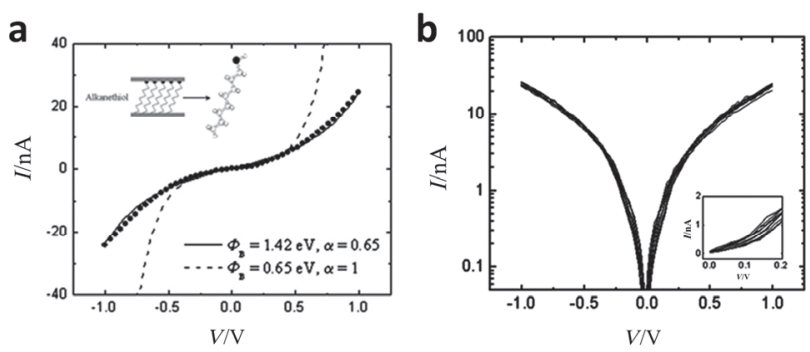

图 11 (a) 十二烷基硫醇 $(C 12) I-V$ 曲线的实验值(圆形符号)和理论值 (实线), 虚线表示从简单的矩形势垒计算出的 $I-V$ 曲线(插图是器件结 构示意图). (b) $\mathrm{C} 12$ 分子结在温度为 300 至 $80 \mathrm{~K}$, 步长为 $20 \mathrm{~K}$ 的 $I-V$ 温度依赖性的对数关系曲线 ${ }^{[64]}$

Figure 11 (a) The C12 I-V data curves that was measured (circular symbols) and calculated (solid curve). Dash line show the calculated $I-V$ from a simple rectangular barrier (illustration is a schematic diagram of the device structure). (b) Temperature-dependent $\log I-V$ curves from 300 to $80 \mathrm{~K}$ with $20 \mathrm{~K}$ steps of $\mathrm{C} 12^{[64]}$
结的电流-电压特性曲线与理论拟合地很好且没有温度 依赖性.

Beebe 和 Frisbie 等 $^{[65]}$ 而后发展了一种分析电流-电 压关系的方法, 即转变电压谱(TVS). 他们发现，当将 $I-V$ 数据重新绘制为图 12 的 $\ln \left(I / V^{2}\right)$ 对 $1 / V$ 时曲线会出现 一个最低拐点. 认为这一拐点是从直接隧穿到 F-N 隧穿 的过渡, 此时势垒从低偏压下的梯形变为高偏压下的三 角形. 此转变电压即势垒高度. 通过 UPS 得到电极费米 能级与分子 HOMO 能级间的势垒偏移, 发现与 TVS 的 转变电压一致.

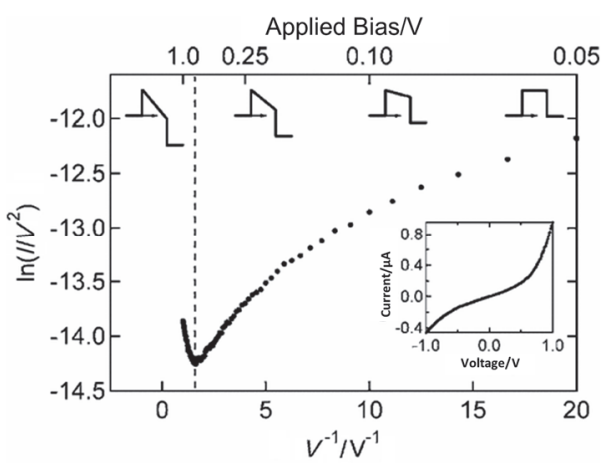

图 12 通过 CP-AFM 测得的 $\mathrm{Au}$-葱硫醇- $\mathrm{Au}$ 结的 100 条 $I-V$ 曲线的平 均值. 插图显示线性的电流-电压数据, 虚线表示转变电压 ${ }^{[65]}$

Figure 12 The average of $100 I-V$ curves of the Au-anthracenethiol-Au junction measured by CP-AFM. The illustration shows current-voltage data on a standard axis. the dotted line indicates the conversion voltage ${ }^{[65]}$

尽管 TVS 曾一度受到广泛应用, 可以很方便地从 $I-V$ 曲线中推断出隧穿势垒. 然而细致的理论表明转变 电压并不是一个真实的物理现象. Vilan 等 ${ }^{[66]}$ 研究发现, TVS 中的拐点只是数学上 $I-V$ 曲线非线性的标度, 任何 非线性的曲线都会在 F-N 图上出现拐点. 然而, 由于隧 穿输运中 $I-V$ 曲线中的非线性的确与隧穿势垒相关联, 即当分子能级接近偏压窗时 $I-V$ 曲线出现明显的非线性. 因此, TVS 仍然可以一定程度上反映隧穿势垒的高度. Baldea $^{[31]}$ 基于单态模型 ${ }^{[67]}$ 给出了转变电压 $V_{\text {trans }}$ 与势垒 高度 $\varepsilon_{\mathrm{h}}$ 之间的关系: $e V_{\text {trans }}=2 \varepsilon_{\mathrm{h}} / \sqrt{3}$. 表明 TVS 依然是 一种有效的分析手段. Jia 等 ${ }^{[68]}$ 利用 TVS 展示了二芳基 乙烯分子结在开态和关态下与电极能级差的不同.

电流一电压特性分析的另一个新发展是基于隧穿机 制的 “通用定律” (law of corresponding states-LCS). Baldea 及其同事 ${ }^{[31]}$ 基于单态模型以及 TVS 给出有关分 子结的 $I-V$ 符合以下关系:

$$
\begin{aligned}
& e V_{\text {trans }}=2 \varepsilon_{\mathrm{h}} / \sqrt{3} \\
& I_{\text {trans }}=G_{1}\left|\varepsilon_{0}\right| \sqrt{3} / e
\end{aligned}
$$

其中 $V_{\text {trans }}$ 是转变电压, $I_{\text {trans }}$ 是转变电流. $I-V$ 曲线分为两 个区域,一个是 $V<V_{\mathrm{C}}$ 的线性区，另一个是 $V>V_{\mathrm{C}}$ 的非 线性区. 再结合这两个公式可以得到如下的一个普遍定 
律: $I_{\mathrm{R}}=I / I_{\mathrm{C}}$

$$
V_{\mathrm{R}}=V / V_{\mathrm{C}}
$$$$
I_{\mathrm{R}}=\frac{2 V_{\mathrm{R}}}{3-V_{\mathrm{R}}^{2}}
$$

这个方程描述了隧穿机制下不同分子结中相似的 $I-V$ 曲线. 实验上, Frisbie 等 ${ }^{[69]}$ 利用 CP-AFM 测得的不同 分子的 $I-V$ 曲线与 LCS 曲线拟合的很好. 为了证明该定 律的普适性，图 13 总结了几个基于 STM 技术测得的 $I-V$ 曲线与 LCS 曲线精确拟合的工作 ${ }^{[29,30,70 \sim 72]}$. 可以看出所 有实验结果与理论曲线相似度都很高.
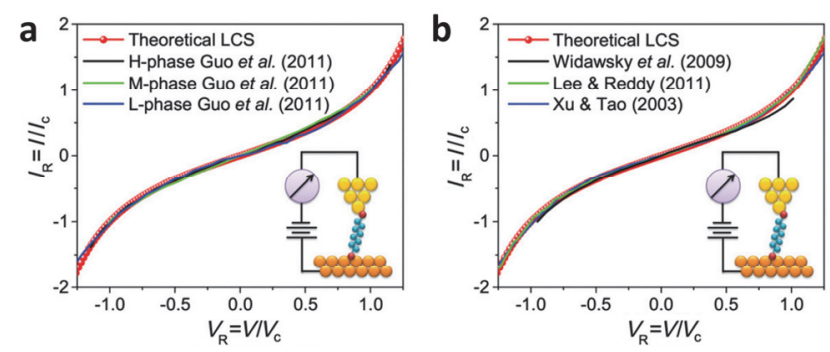

图 13 (a) 分别为 $2151,1661,1661$ 条 $I-V$ 曲线的平均值, 其中 $\mathrm{H}, \mathrm{M}, \mathrm{L}$ 分别为金电极与辛二硫醇 (C8DT) 分子结的高、中、低电导. (b) 测得 的 STM-BJ 技术中金电极与对 4,4'-二氨基花, C $8 \mathrm{DT}$ 和 $44 \mathrm{bpy} \equiv 4$ 相连 的单个 $I-V$ 曲线, 欧姆电阻 $R$ 在三个数量级上变化 $[R \approx 1.3 \mathrm{M} \Omega$ 到 $R \approx$ $1.2 \mathrm{G} \Omega]^{[29,30,70 \sim 72]}$

Figure 13 (a) Averages of 2151, 1661, $1661 \mathrm{I}-\mathrm{V}$ traces for the high(H); medium- (M); low- (L) conductance phases of octanedithiol (C8DT) junctions with gold electrodes and (b) In the measured STM-BJ technique, the gold electrode is connected to a single $I-V$ curve connected to $4,4^{\prime}$-diaminopurine, C8DT and $44 \mathrm{bpy} \equiv 4$, and the ohmic resistance $R$ varies in three orders of magnitude $[R \approx 1.3 \mathrm{M} \Omega \text { to } R \approx 1.2 \mathrm{G} \Omega]^{[29,30,70 \sim 72]}$

\section{2 跳跃机理}

跳跃机理是另一种主要的电荷输运行为, 通常发生 在较长分子中. 在跳跃机制中, 电荷首先到达分子再传 输到另一侧电极. 因此电荷在传输过程中会与分子发生 相互作用, 分子的形变以及周围环境的极化会暂时性捕 获电荷, 再通过热激活使电荷继续传输. 因此, 分子结 电导与温度有关, 并遵循 Arrhenius 公式:

$$
G \propto \exp \left(\frac{-E_{\mathrm{a}}}{k_{\mathrm{B}} T}\right)
$$

其中 $k_{\mathrm{B}}$ 是玻尔兹曼常数, $T$ 是温度, $E_{\mathrm{a}}$ 是活化能, 即电导 与温度呈指数相关. 在对隧穿机制的介绍中提到其电导 没有明显的温度依赖性，而跳跃机制与温度有关，因此， 是否具有温度依赖性可成为判断隧穿和跳跃两种输运 机理的依据. 但在一定条件下, 两种传输机理可以相互 转变.

关于跳跃机制的温度依赖性, Chen 等 ${ }^{[73]}$ 利用自组装 技术制备了二异氧酸酯分子结, 并在不同温度下测得了 分子结电导. 如图 14 所示, 可以观察到在不同偏压下分 子结电导均有明显的温度依赖性. Selzer 等 ${ }^{[74]}$ 利用电迁 移技术制备了 $\mathrm{Au}$-苯基乙炔基-4'-巯基- $\mathrm{Au}$ 分子结, 并观
察到高温下电流随着 $1 / T$ 呈指数衰减且从温度依赖转变 成低温下的温度不依赖, 即电荷传输机理由跳跃转变成 了隧穿(图 15).

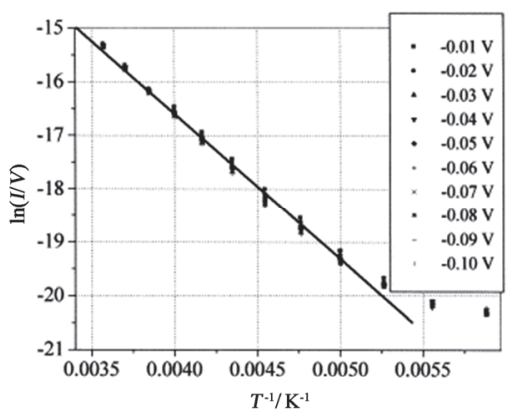

图 14 在大于 $-0.1 \mathrm{~V}$ 的偏压下, Pd-SAM-Pd 分子结的 $\ln (1 / V)$ 和 $1 / T$ 的曲线图 ${ }^{[73]}$

Figure 14 Pd-SAM-Pd junction $\ln (1 / V)$ and $1 / T$ curves at biases larger than $-0.1 \mathrm{~V}^{[73]}$
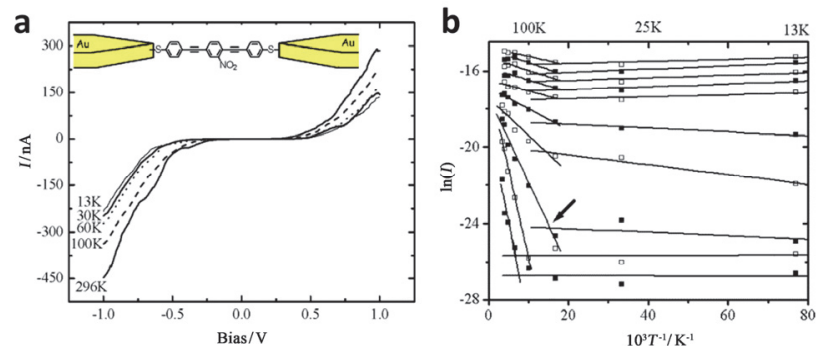

图 15 (a) 同一个分子结在几个温度下测量的 $I-V$ 曲线, 插图是金属分子-金属结的示意图. (b) 在不同偏压下的 $\ln (I)$ 对 $1 / T$ 的 Arnhenius 点的曲线图, 显示了电导由低温下的温度不依赖转变为高温下的温度 依赖. 箭头显示从温度不依赖到热激活过程的转折点 ${ }^{[74]}$

Figure 15 (a) $I-V$ curves measured on one of the junctions for several temperatures. Inset is the schematic of metal-molecule-metal junction. (b) Arrhenius plots of $\ln (I)$ vs $1 / T$ at different bias voltages showing a transition in conductance from temperature independent at low temperature range and temperature dependent at high temperature. The arrow shows the turning point from temperature-independent to thermal activated process $^{[74]}$

跳跃的另一个特点是电导随长度线性衰减 ${ }^{[25]}$. 当分 子的长度逐渐增加时, 可观察到从隧穿到跳跃的转变. Frisbie 等 ${ }^{[63]}$ 在金基底上生长不同长度(范围从 1.5 到 7.3 $\mathrm{nm}$ )的共轭低聚亚苯基亚胺(OPI)分子, 利用 CP-AFM 技 术进行 $I-V$ 测试, 得到了电阻与分子长度的关系图, 如 图 16 所示. 可观察到随着分子长度的增加传输机理明 显的转变. 长度 $<4 \mathrm{~nm}$ 时, 电导呈指数衰减, 可以用公 式 $I=I_{0} e^{-\beta d}$ 很好地拟合, 衰减因子 $\beta$ 为 $3.0 \pm 0.08 \mathrm{~nm}^{-1}$. 长度 $>4 \mathrm{~nm}$ 时, 电导呈线性衰减, $\beta=0.9 \pm 0.07 \mathrm{~nm}^{-1}$. 此外, 长分子电导还具有很强的温度依赖性, 而短分子 电导是温度不依赖的. 由此表明电荷输运机理从短分子 的隧穿转变为长分子的跳跃. 相同结论 Choi 等 ${ }^{[75]}$ 也报 道过.

近些年来跳跃传输在生物分子体系受到了广泛的 关注,一方面是为了帮助研究生命体系内的电荷转移, 另一方面是探索生物分子作为分子电子器件的功能单 

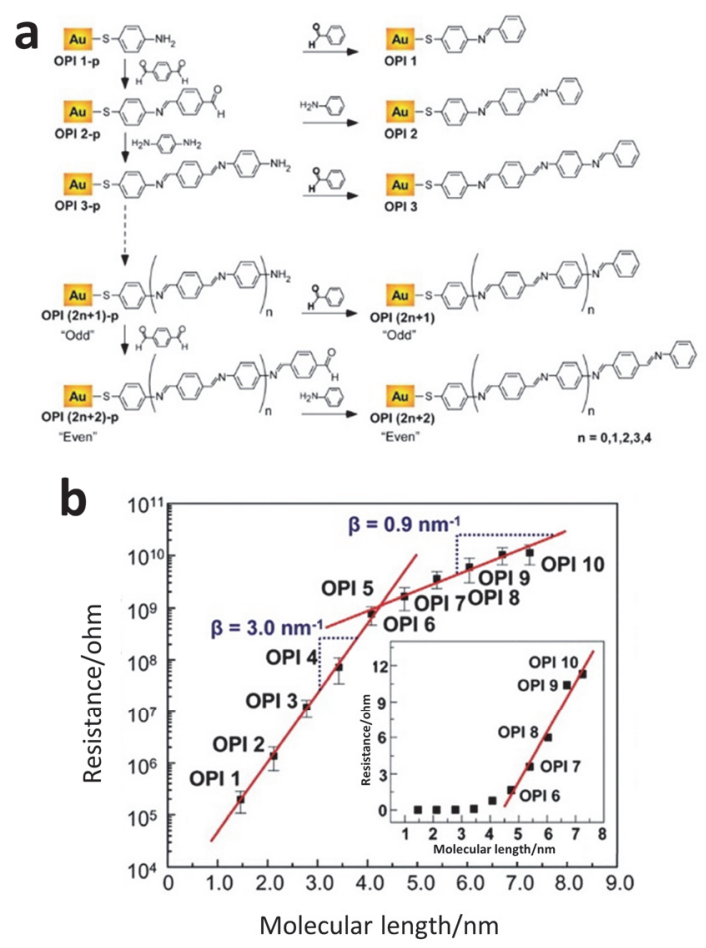

图 16 (a) 不同长度的 OPI 分子. (b) 通过 CP-AFM 测量的 OPI 结的 电阻 $(R)$ 与长度 $(L)$ 的半对数图 (插图为 $R$ 与 $L$ 的线性图) ${ }^{[63]}$

Figure 16 (a) The synthetic route of various length OPI wires. (b) Semilog plot of $R$ versus $L$ for the OPI junctions measured by CP-AFM. (Inset a linear plot of $R$ versus $L)^{[63]}$

元. 最典型的是 DNA，其双螺旋结构中的碱基对形成了 共轭分子面对面排列的分子管 ${ }^{[76]}$, 很早就被认为可能是 纳米尺度的电荷传输通路 ${ }^{[77,78]}$. 对 DNA 电荷传输的机 理的研究也非常丰富 ${ }^{[79]}$, 通常认为与有机分子类似, 较 短链的是隧穿传输占主导, 长链的跳跃占主导. 最新的 研究主要关注于两种机理之间的转变过程及其化学本 质. Tao 等 ${ }^{[80]}$ 利用 STM-BJ 技术测试了含有堆叠 $\mathrm{G}$ 碱基 以及交替 $\mathrm{G}$ 碱基的两种短链 DNA 分子结电导. 得到了 不同序列中的电导随长度的变化, 如图 17 所示. 从图中 可以发现, 交替 $\mathrm{G}$ 碱基序列中电阻随长度线性增加, 说 明其电荷传输是跳跃机制[6]. 而堆叠 $\mathrm{G}$ 碱基序列中电阻 在随长度线性增加的过程中出现了振荡叠加的现象. 这 些振荡与金原子链中的类似, 因此, 认为是隧穿机制的 特征. 然而, 与纯的隧穿不同的是其振幅随长度迅速衰 减. 所以, 这些振荡被解释为是隧穿与跳跃的中间传输 机制的标志.

另一个近些年被广泛研究的体系是蛋白. 生命体系 中的能量转化和信号传递相关的电荷转移过程都有蛋 白参与, 同时, 跨越蛋白的电荷转移都是长程的. 因此 弄清蛋白中电荷传输的机理(隧穿 Vs 跳跃)也特别重 要 ${ }^{[81]}$. Cahen 等 ${ }^{[82]}$ 通过掺杂的方法研究了天然人血清白 蛋白(HSA)和人造色素 $(\mathrm{CytC})$ 电导的温度依赖性, 实验 结果如图 18 所示. 从图中可以得到以下几个信息: (1) 两种蛋白的传输机制(温度依赖性)很相似; (2) 在给定
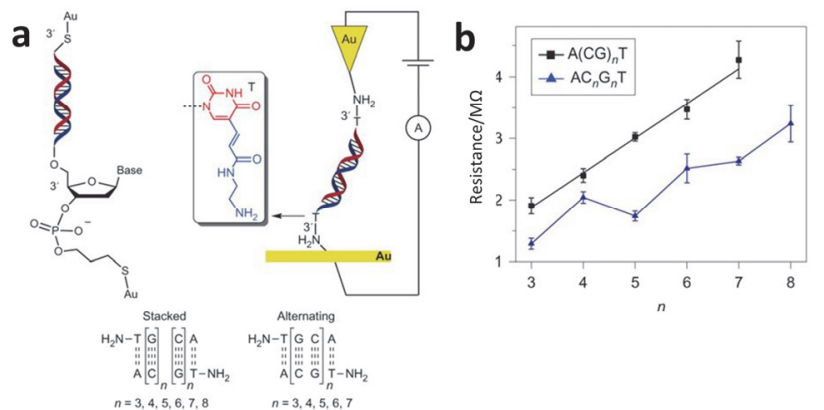

图 17 (a) DNA 分子通过糖(左)及胸腺嘧啶碱基(右)连接到两端电极. $\mathrm{A}(\mathrm{CG})_{n} \mathrm{~T}$ 和 $\mathrm{AC}_{n} \mathrm{G}_{n} \mathrm{~T}$ 分别表示交替和堆叠的 $\mathrm{G}$ 序列. (b) 交替(黑色方 块)和堆叠(蓝色三角形)的 DNA 序列的电阻与序列中 CG 数量的关 系 ${ }^{[80]}$

Figure 17 (a) DNA molecules connected to two electrodes via the sugar (left) and directly via the thymine base (T, right), $\mathrm{A}(\mathrm{CG})_{n} \mathrm{~T}$ and $\mathrm{AC}_{n} \mathrm{G}_{n} \mathrm{~T}$ denote alternating and stacked $\mathrm{G}$ sequences, respectively. (b) Resistance of alternating (black squares) and stacked (blue triangles) DNA sequences versus the number of CG in the sequences ${ }^{[80]}$

偏压下，随着 HSA 与掺杂物维甲酸(RA)比例的增加温 度不依赖区的电流值增加，在温度依赖区随掺杂比的增 加活化能降低, 说明掺杂物对蛋白中的电荷传输机理产 生了很强的影响；(3) 在足够低的温度下，电导表现出 温度不依赖的行为, 在某较高温度下电导表现出温度依 赖的行为, 说明两种不同的传输机制共存. 在低温下主 要机制是隧穿，而较高温度下主要是跳跃.

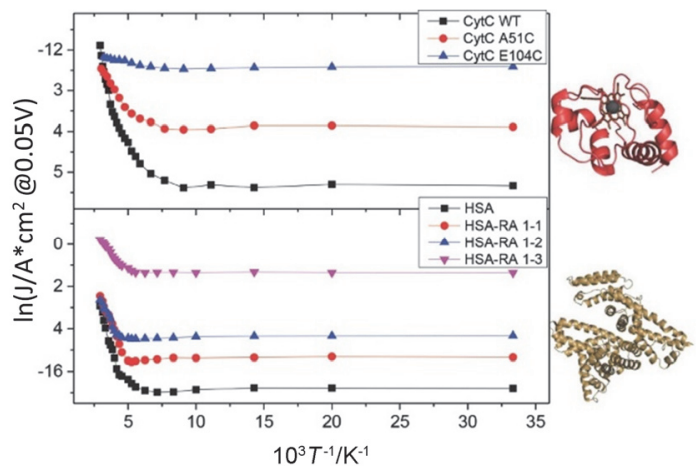

图 18 两种蛋白 $\mathrm{HSA}$ (褐色), $\mathrm{CytC}$ (红色)的电流随温度变化的关系 图 $^{[82]}$

Figure 18 Relationship between conductance of two proteins HSA (brown) and $\mathrm{CytC}$ (red) as a function of temperature ${ }^{[82]}$

隧穿与跳跃机制作为微观尺度下的两种主要传输 机理，它们各自的特点已经比较清楚. 近些年的研究更 多关注于跳跃到隧穿之间的转变过程. 目前尚无完整的 输运理论可以完整涵盖隧穿和跳跃传输. 也没有研究可 以详细揭示两者转变的精细化学基础. 此外, 跳跃传输 的基础是分子热运动, 与此相关的分子结的热性质, 如 热电势、热导等也是研究的热点问题.

\section{3 基于量子干涉的电荷输运及应用}

量子干涉效应 $(\mathrm{QIE})$ 是近些年备受关注的一种输运 现象, 是分子特有的量子输运特性. 电荷传输中的量子 干涉效应源于电子在相干传输中通过不同分立的分子 
轨道( $\mathrm{MO})$, 从而形成的波函数的干涉效应. 在分子电子 学领域, QIE 首先由 Ratner 等 ${ }^{[83]}$ 对线性共轭分子蒽(AC), 交叉共轭分子葸醌(AQ), 具有破坏性共轭结构的分子 二氢葱酮 $(\mathrm{AH})$ 的三种分子进行理论计算时发现. 这三 种长度相同的分子中, $\mathrm{AQ}$ 分子的透射谱在电极费米能 级附近由于破坏性量子干涉表现出显著下降.

Hong 等 ${ }^{[84]}$ 利用 MCBJ 技术对以上三种分子结进行 了电学特性的表征, 得到了如图 19 所示的电导变化规 律. 从图中可以观察到 $\mathrm{AQ}$ 分子电导比 $\mathrm{AC}$ 低约 300 倍, 这表明, 与 $\mathrm{AC}$ 相比, $\mathrm{AQ}$ 的交叉共轭确实具有较低的电 导, 这与预测 $\mathrm{AQ}$ 中存在破坏性量子干涉(DQI)的理论 计算相一致. 这是在单分子水平上第一个观察到 DQI 现 象的实验之一. 之后实验上通过 $\mathrm{EGaIn}^{[85]}, \mathrm{CP}-\mathrm{AFM}^{[86]}$ 及顶电极蒸镀等 ${ }^{[87]}$ 方法也都测得了上述三种分子的 SAM 电导, 也发现 AQ 分子的电流密度最低.

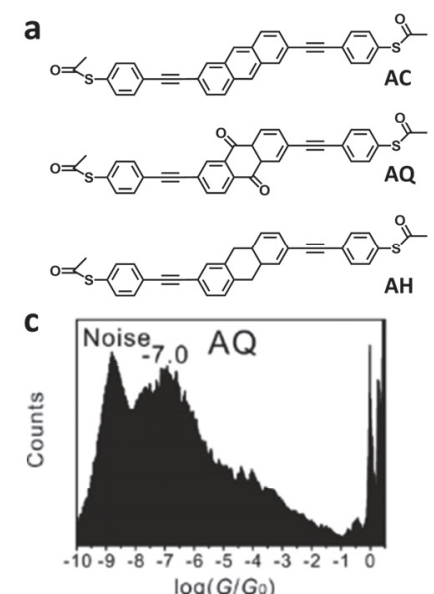

$\log \left(G / G_{0}\right)$

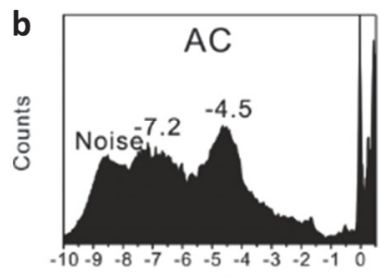

$\log \left(G / G_{0}\right)$

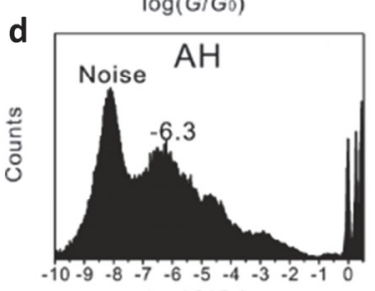

$\log \left(G / G_{0}\right)$
图 19 (a) $\mathrm{AC}, \mathrm{AQ}, \mathrm{AH}$ 分子结构, (b) $\mathrm{AC}$ 分子的 500 条曲线的一维电 导直方图, (c) $\mathrm{AQ}$ 分子和(d) $\mathrm{AH}$ 分子 ${ }^{[84]}$

Figure 19 (a) Molecular structures of AC, AQ, and AH, (b) 1-D conductance histograms constructed from 500 individual traces of $\mathrm{AC}$, (c) of $\mathrm{AQ}$ and (d) of $\mathrm{AH}^{[84]}$

量子干涉效应通常与分子结构有关, 因此 Hong 等 ${ }^{[88]}$ 利用 MCBJ 和 STM-BJ 技术研究了一系列的带有吡 啶针定基团的低聚物(亚苯基亚乙炔基一OPE)的单分子 电导. 发现针定基团中氮原子取代基的位置仅对单分子 电导有微弱影响, 但中心环的位置对电导有较强影响. 类似的工作还有通过改变 OPE 分子中心环中氮原子的 取代位置调节 DQI, 进而调节单分子结电导 ${ }^{[89]}$. 该研究 结果为调整单分子结中的量子干涉效应提供了指导, 对 于设计具有理想功能的分子器件至关重要.

近两年, 有关 QIE 在分子电子学领域的应用也逐渐 兴起, Hong 和 Zhang 等 ${ }^{[90]}$ 利用 MCBJ 技术基于量子干 涉效应实现了在单分子水平上对 SDPP 和 SPPO 异构体 的识别. 理论上 SPPO 去质子化时, 有两个可能的共轭 结构, 分别是线性共轭和交叉共轭. 线性共轭与其本身 类似, 能提供连续的电荷传输; 交叉共轭有两个单-双 交替路径没有相互共轭, 因此会出现电导低的现象. 基
于此设计了相关实验，如图 20 所示. 实验结果显示，可 根据典型的电导图差异识别 SDPP 和 SPPO 异构体. 这 项工作首次在实验上获得了单分子水平识别异构体的 方法, 为异构体识别和隔离提供了一种新的、有效的方 法以及为新分子器件的构筑提供了可能.
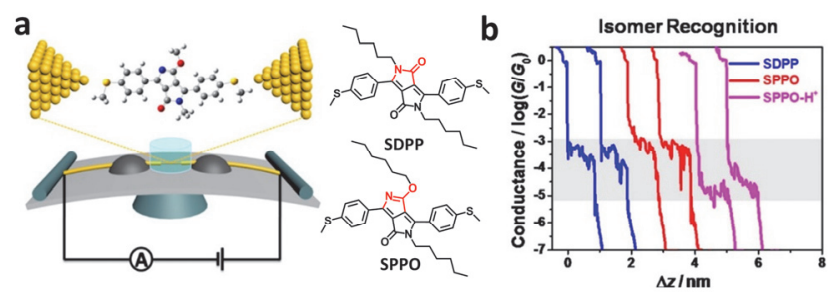

图 20 (a) MCBJ 装置和两个 DPP 异构体的示意图. (b) SDPP, SPPO 和 $\mathrm{SPPO}-\mathrm{H}^{+}$的典型电导曲线 ${ }^{[00]}$

Figure 20 (a) Schematic of MCBJ setup and the two DPP isomers. (b) Typical measured conductance distance traces of SDPP, SPPO and SPPO- $\mathrm{H}^{+[90]}$

Hong 等 ${ }^{[91]}$ 利用电化学栅极调控的方法结合 MCBJ 技术对噻吩类单分子结的电导进行了调控. 此研究是基 于量子干涉原理, 通过改变电化学栅极电压来改变含有 破坏性量子干涉(DQI)的分子结电导, 使得电导可实现 两个数量级的改变. 实验结果显示不含 DQI 的分子电导 随栅极电压的改变仅仅是 8 倍, 如图 21 所示. 证明了电 化学栅极调控是对基于量子干涉的分子器件电学特性 原位调控的有效手段. 电导的较大变化, 为基于 DQI 构 建分子开关器件提供了可能. 另外, Hong 等 ${ }^{[92]}$ 最新的综 述中对单分子结电荷输运中的 QIE 的识别、QIE 的调控 及其潜在应用等都做了全面而详细的总结.
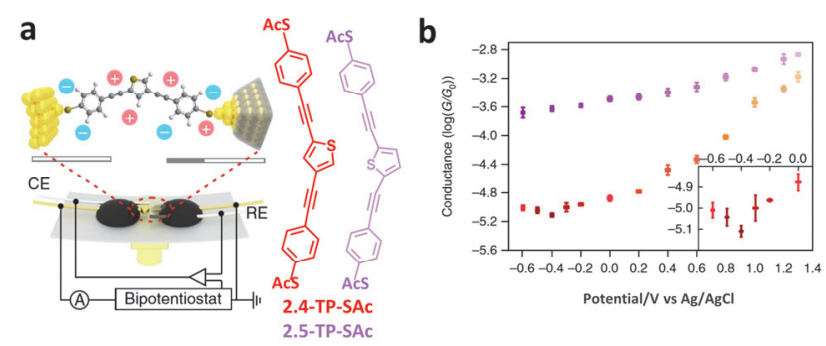

图 21 (a) 电化学栅极调控结合 MCBJ 技术制备的具有硫代乙酰基针 定基团的噻吩衍生物单分子结示意图. (b) 栅极电位从 $0.6 \sim 1.3 \mathrm{~V}$ 时 2,5-TP-SAc(紫色)和 2,4-TP-SAc(橙色)分子结电导的变化(插图显示电 位从 $-0.6 \sim 0 \mathrm{~V}$ 的方法 ${ }^{[91]}$

Figure 21 (a) Schematics of the electrochemically gated MCBJ technique and molecular structures of thiophene derivatives with anchoring groups of thioacetyl (-SAc). (b) Tendency of the molecular conductance of 2,5-TP-SAc (purple) and 2,4-TP-SAc (orange) versus electrode potentials from $-0.6 \mathrm{~V}$ to $1.3 \mathrm{~V}$. Inset, magnification from $-0.6 \mathrm{~V}$ to $0 \mathrm{~V}^{[91]}$

\section{4 分子器件的应用}

分子电子学的诞生承载着人们对分子级微型器件 的梦想. 如今, 分子电子器件的发展, 已经远远超越了 最初取代无机半导体的传统功能的器件, 新功能器件的 发明层出不穷. 


\section{1 分子整流器}

整流器是一种单向导电的电子器件, 普通的固体整 流器一般基于半导体的 $\mathrm{p}-\mathrm{n}$ 结 ${ }^{[3]}$. 对于分子级别的器件 要实现整流特性, 也要有与 $\mathrm{p}-\mathrm{n}$ 结相似的性质. 早期 Ratner ${ }^{[2]}$ 有关分子整流器的设想是基于类似 $\mathrm{p}$-n 结的有 机给-受体分子. 最初的分子整流器设计是基于 Ratner 的想法，即将给-受体分子置于分子结中.

Sita 等 ${ }^{[94]}$ 利用自组装技术制备了金属-分子-金属结 的分子整流器, 探究了金银两种基底对整流特性的影 响. 发现银作为基底时, 整流现象更显著. 并对整流现 象产生的原因进行研究, 发现整流特性源于施加偏压 后, 分子与两电极间势垒高度的不对称. 对此类自组装 分子结器件的电子行为有了新的认识. Metzger 等 ${ }^{[95]}$ 利 用金属-LB 膜-金属的结构测得了 $\mathrm{C}_{16} \mathrm{H}_{33}-\mathrm{Q}_{3} \mathrm{CNQ}$ 分子的 整流特性. 为后续研究分子整流器更深层次的机理提供 思路.

Tao 等 ${ }^{[96]}$ 使用 STM-BJ 技术来研究通过选择性去保 护合成的与联苯分子相同主体不同端基的二嘧啶基二 苯基分子结器件的整流特性与分子对称性以及取向之 间的关系. 实验中为确保在形成单分子结的过程中, 分 子的取向不变, 对 STM 装置的压电元件额外施加了一 个频率为 $2 \mathrm{kHz}$ 的正弦交流电, 可以起到对针尖与金基 底的距离有 $0.02 \sim 0.08 \mathrm{~nm}$ 的调制作用. 通过电流的振 幅随时间的变化监测针尖与电极的距离, 从而形成分子 结. 实验中发现, 非对称的二嘧啶基二苯基分子结器件 具有明显的整流特性, 整流比为 $5( \pm 1.5 \mathrm{~V})$. 而对称的 联苯分子没有出现整流效果, 如图 22 所示. 为了研究其 出现整流现象的具体原因, 通过理论计算得知在施加正 偏压时, 由于空穴的基态波函数的非对称性导致其从针 尖转移到分子的概率增加, 从而出现整流.
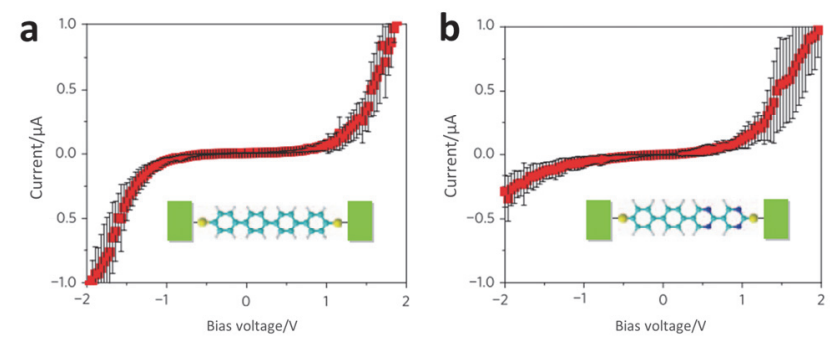

图 22 (a) 对称分子结器件的 $I-V$ 曲线. (b) 非对称分子的 $I-V$ 曲线 ${ }^{[96]}$ Figure 22 (a) Current-voltage $(I-V)$ curves for Symmetrical molecular junction device. (b) Current-voltage $(I-V)$ curves for the non-symmetric molecule ${ }^{[96]}$

此外, 界面处的耦合也会引起整流. 其中, 包括不 同基团与电极的耦合. Kushmerick 等 ${ }^{[9]}$ 在实验上证实了 通过改变针定基团使得分子/电极界面的耦合不同可以 实现整流. Wang 等 ${ }^{[98]}$ 利用 STM-BJ 技术, 选择末端为硫 醇和氨基两种针定基团的分子在理论和实验上再次证 实了这一观点.

Venkataraman 等 ${ }^{[99]}$ 利用 STM-BJ 技术提出了一种新
的整流机制. 采用一端为固定的金-碳键连接，另一端 为不同端基与金电极相互作用制备多种分子结器件，如 图 23 所示. 室温下对其进行 $I-V$ 测试, 发现零偏压下器 件有较高的电导, 低偏压下, 器件出现了整流特性, 平 均整流比为 $1.7( \pm 0.8 \mathrm{~V})$. 对不同分子结的测试结果进 行比较发现: 分子/电极界面耦合强度最弱的分子整流 比最大, 反之, 整流比最小. 与 DFT 理论计算相一致. 该结果表明, 可以通过设计分子调控整流比. 为促进未 来器件设计以及优化器件性能提供依据.
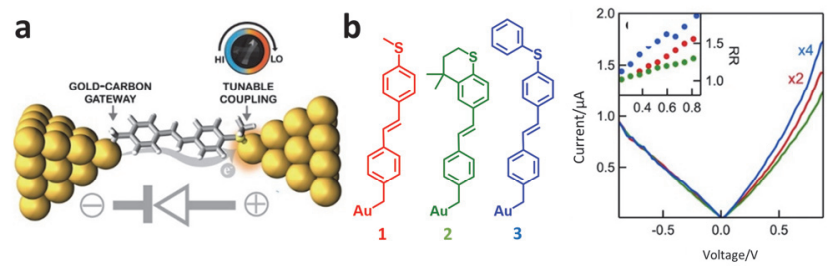

图 23 (a) STM-BJ 形成的分子结器件. (b) 三种分子的结构以及得到 的整流特性 ${ }^{[99]}$

Figure 23 (a) Molecular junction device formed by STM-BJ. (b) The structure of the three molecules and the resulting rectification characteris$\operatorname{tics}^{[99]}$

近些年来, 人们发现, 当电活性基团位于分子结中 并与两电极相对位置不对称时, 由于分压效应，可获得 较高的整流比. Whitesides 等 ${ }^{[100]}$ 将带有二茂铁头基的烷 基硫醇分子自组装在超平银基底上, EGaIn 作为顶电极 制备了分子结器件, 对其进行 $I-V$ 测试得出以下结论: (1) 当二茂铁的 HOMO 能级处于相对两端电极不对称 的状态并且能量低于两电极的费米能级时可实现整流; (2) 只有一个轨道 (HOMO 或 LUMO) 能级与电极费米能 级相近是获得高整流比的必要条件. 基于此, 该组 ${ }^{[101]}$ 利 用同样的器件结构, 通过施加正反偏压改变二茂铁的电 荷输运机理而获得了大于 100 的整流比.

此后, Nijhuis 等 ${ }^{[102]}$ 对制备工艺进行了改进, 利用较 长烷基链和二茂铁的分子结构以及用铂代替银来增强 击穿电压. 并且两个二茂铁单元被炔基分开, 使得两个 轨道 $\mathrm{HOMO}$ 和 $\mathrm{HOMO}-1$ 都可以在低偏压下进入导电 窗而得到了整流比为 $10^{5}$ 的分子整流器, 如图 24 所示. 这种分子整流器未来可应用于超高速电子器件或纳米 级器件中.

\section{2 分子开关}

对于某种特定材料的器件, 施加偏压, 当场强达到 一定值时, 器件可由绝缘态 (0)转为导电态 (1); 通过某 种刺激(如反向电场、脉冲电流、光或热等)又可使器件 由 1 态恢复到 0 态, 这种器件称之为开关器件 ${ }^{[81]}$. 某些 特定的分子也有这种开关特性, 例如具有光响应的噻 吩、吡咯以及二芳基乙烯分子等.

Feringa 等 ${ }^{[103]}$ 将二芳基乙烯分子自组装在金基底上 制备分子层器件，利用不同波长的紫外光照射，使其实 现开关效应. Matsuda 等 ${ }^{[104]}$ 将二芳基乙烯二硫代苯酚吸 

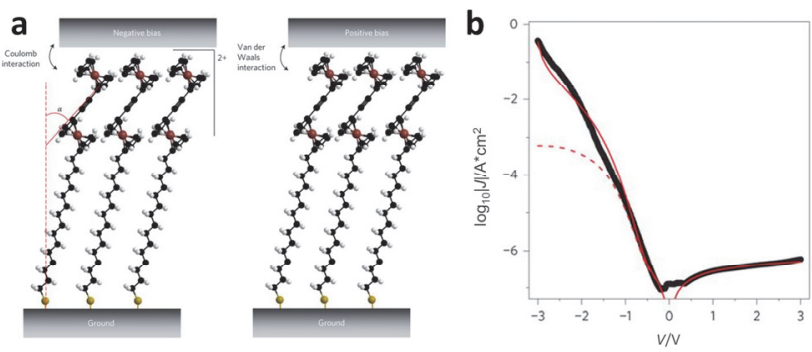

图 24 (a) EGaIn-HSC ${ }_{15} \mathrm{Fc}-\mathrm{C} \equiv \mathrm{C}-\mathrm{Fc}-\mathrm{Pt}$ 分子结器件的结构. (b) 在 $\mathrm{Pt}$ (黑 色线)上的分子二极管的 $\log 10|\mathrm{~J}|(\mathrm{V})$ 曲线在有(红色实线)和没有(红色 虚线)偏压依赖时与理论的拟合 ${ }^{[102]}$

Figure 24 (a) Structure of $\mathrm{HSC}_{15} \mathrm{Fc}-\mathrm{C} \equiv \mathrm{C}-\mathrm{Fc}$ molecular junction device. (b) $\log 10|\mathrm{~J}|(\mathrm{V})$ curve of the molecular diode on Pt (black dots) with fits to with (solid red line) and without (dashed red line) the functional $\mathrm{n}(\mathrm{V})^{[102]}$

附在金纳米粒子上制备分子器件, 通过紫外和可见光照 射使其实现开关效应. 但是，由于分子与金的强耦合对 分子开态的光响应有淬灭作用, 因此器件不能在开关态 下自由切换 ${ }^{[103]}$.

为了制得可切换的开关器件, Guo 等 ${ }^{[105]}$ 采用在单壁 碳纳米管 $(\mathrm{SWNT})$ 上修饰二芳基乙烯分子的方法, 利用 在紫外和可见光刺激下其非共轭(开态)及共轭(关态)结 构的相互转换实现可以切换的开关效应, 如图 25 所示. 为开关器件的制备提供了一定的参考; 但是不足之处在 于器件性质不稳定, 开关比不高.
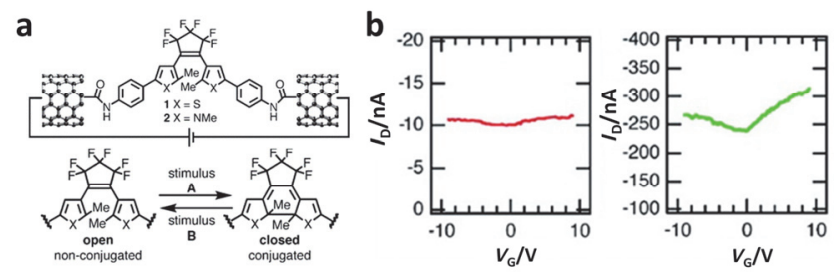

图 25 (a) 单个 SWNT 电极末端之间的分子桥以及在共轭和非共轭 的分子结构之间切换示意图. (b) 单独的开态 SWNT 器件以及经 UV 照射后器件变为关态 ${ }^{[105]}$

Figure 25 (a) Molecular bridge between the ends of a single SWNT electrode and switching between conjugated and non-conjugated molecular structures. (b) Schematic diagram of a separate open-state SWNT device and the device becomes off after UV illumination ${ }^{[105]}$

之后, Guo 等 ${ }^{[106]}$ 将光致发光的同分异构体分子与石 墨烯电极相连, 制备了分子开关. 通过合理设计侧链 (引入亚甲基)和针定基团(引入氟单元)降低了分子与电 极之间的耦合, 实现了稳定可切换的分子开关器件, 如 图 26 所示. 这个研究为将分子电子器件整合到电子电 路中提供了可能, 但开关比仅在几十左右.

最近, Guo 等 ${ }^{[107]}$ 利用在石墨烯上修饰二芳基乙烯分 子, 在低温下通过紫外和可见光的照射使器件具有稳定 可自由切换的开关特性，如图 27 所示，达到了当时最高 的开关比 100. 这个结果表明具有一定导电性并且能固 定在固态电极上的分子可以成为未来纳米电子学的重 要组成部分. a
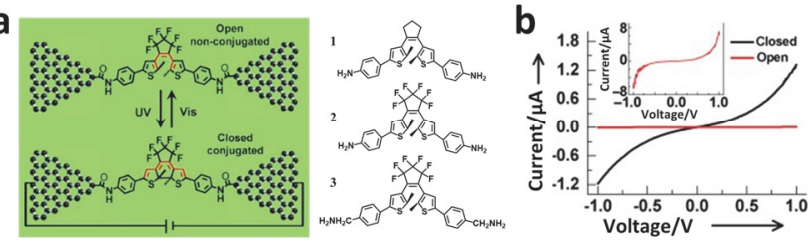

图 26 (a) 石墨烯-二芳基乙烯-石墨烯结的开关态切换及分子结构. (b) $V_{\mathrm{G}}=0 \mathrm{~V}$ 时的开态(红线)和关态(暗线)的 $I-V$ 特性，插图显示了开态 的放大 $I-V$ 曲线 ${ }^{[106]}$

Figure 26 (a) Switching of graphene-diarylethene junctions and molecular structure. (b) $I-V$ characteristics of open (red line) and closed (dark line) states at $V_{\mathrm{G}}=0 \mathrm{~V}$. The inset shows the enlarged $I-V$ curve for the open state ${ }^{[106]}$
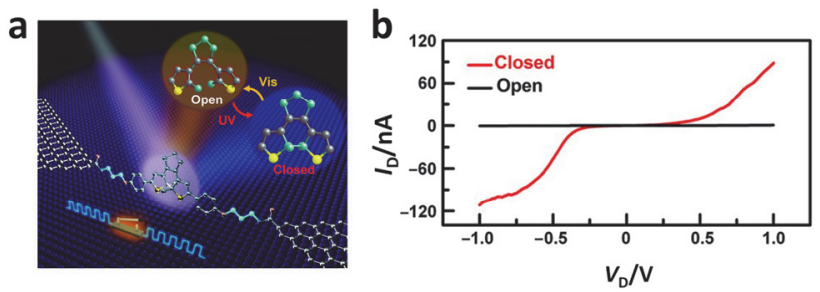

图 27 (a) 石墨烯-二芳基乙烯-石墨烯分子结的示意图, 明亮处是通 过亚甲基连接的分子桥. (b) 栅极电压 $V_{\mathrm{G}}=0 \mathrm{~V}$ 时单个二芳基乙烯分 子结的开态(黑线)和关态(红线)的 $I-V$ 特性. $V_{\mathrm{D}}$ 是漏极电压, $I_{\mathrm{D}}$ 是漏极

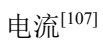

Figure 27 (a) Schematic of a graphene-diarylethene-graphene junction that highlights the expansion of the molecular bridge by methylene groups. (b) $I-V$ characteristics of individual diarylethenes in open (black line) and closed (red line) format gate voltage $V_{\mathrm{G}}=0 \mathrm{~V} . V_{\mathrm{D}}$ is drain voltage; $I_{\mathrm{D}}$ is drain current ${ }^{[107]}$

\section{3 分子晶体管}

场效应晶体管(FET) 是一种通过栅极调控实现源漏 电极的接通和断开的三端器件. 在分子电子学领域, 起 初大多数的研究都集中于双端分子器件. 然而, 像 FET 这样的三端分子器件在实际中却是迫切需要的, 因为它 是集成电路的基本元件. 最初在碳纳米管 ${ }^{[108]}$ 和纳米 线 ${ }^{[109]}$ 中证明了类似 FET 的行为, 理论模型也预测了单 个分子的电导可以通过常规的 FET 栅极调控来改变 ${ }^{[110]}$.

$\mathrm{Xu}$ 等 ${ }^{[111]}$ 利用电化学作为栅极, 硫醇分子自组装的 金电极作为源漏电极制备了 $\mathrm{n}$-型分子晶体管, 在室温下 可通过栅极调控使源漏电流有三个数量级的改变, 如图 28 所示. Lee 等 ${ }^{[112]}$ 利用光刻技术制备的金电极作为源漏 电极, 用铝作为栅极首次实现了三端固态分子晶体管, 并可实现在 $4.2 \mathrm{~K}$ 低温下通过栅极调控分子轨道与源漏 电极的共振耦合来改变载流子的传输机制, 如图 29 所 示. 为以后电子器件的设计及研究载流子传输机制提供 了参考，但该晶体管只能在低温下才可实现栅极调控的 作用.

Zant 等 ${ }^{[113]}$ 在分子晶体管的制备工艺上做了研究, 通过化学反应以及电灼的新方法制备出用几层石墨烯 连接栅极的分子晶体管, 器件的制备及性质, 如图 30 所 示. 此晶体管在高温下也比较稳定，同时可实现通过栅 极的调控改变分子结电导. 由于石墨烯电极具有很好的 


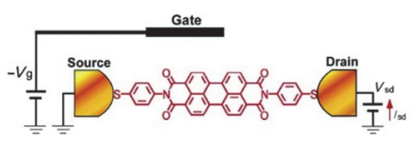

b

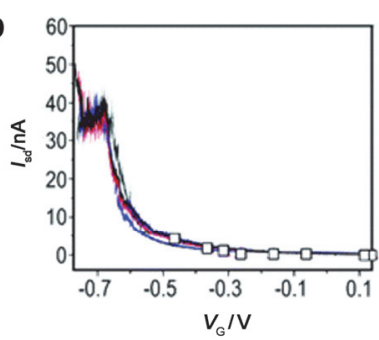

图 28 (a) 具有电化学栅极的单分子晶体管的示意图. (b) 单个 PTCDI 分子晶体管的源极-漏极电流 $\left(I_{\mathrm{sd}}\right)$ 与栅极电压 $\left(V_{\mathrm{g}}\right)$ 的关系, 从电 导直方图的峰值位置获得空心方块, 通过在扫描栅极电压的同时直接 记录源极电流获得实线 ${ }^{[111]}$

Figure 28 (a) Schematic of a single molecule transistor with an electrochemical gate. (b) Source-drain current $\left(I_{\mathrm{sd}}\right)$ versus gate voltage $\left(V_{\mathrm{g}}\right)$ for a single PTCDI molecule transistor. The open squares were obtained from the peak position of the conductance histograms. The solid lines were obtained by directly recording the sourcedrain current while sweeping the gate voltage $\mathrm{e}^{[111]}$

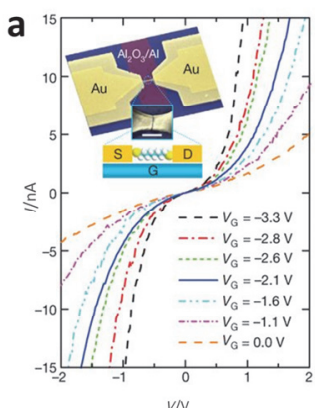

b

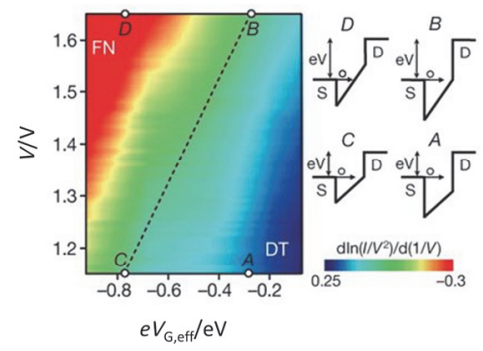

图 29 (a) 在 $4.2 \mathrm{~K}$ 温度下对于不同的 $V_{\mathrm{G}}$ 值测得的具有代表性 $I-V$ 曲 线, 插图显示了器件结构和原理图, 其中 $\mathrm{S}$, 源极, $\mathrm{D}$, 漏极, $\mathrm{G}$, 栅极, 比例尺: $100 \mathrm{~nm}$. (b) $\operatorname{dn}\left(I / V^{2}\right) / \mathrm{d}(1 / V)$ 的二维彩色图, 显示出了对应于四 个不同区域(点 $\mathrm{A} \sim \mathrm{D}$ ) 的能带图, F-N 隧穿到 DT(直接隧穿 $)^{[112]}$

Figure 29 (a) Representative $I-V$ curves measured at $4.2 \mathrm{~K}$ for different values of $V_{\mathrm{G}}$. Inset, the device structure and schematic. S, source; D, drain; G, gate. Scale bar, $100 \mathrm{~nm}$. (b) Two-dimensional colour map of $\mathrm{d} \ln \left(\mathrm{I} / V^{2}\right) / \mathrm{d}(1 / V)$ (from Fowler-Nordheim plots). Energy-band diagrams corresponding to four different regions (points $\mathrm{A} \sim \mathrm{D}$ ) are also shown. F-N tunnelling; DT, direct tunneling ${ }^{[112]}$
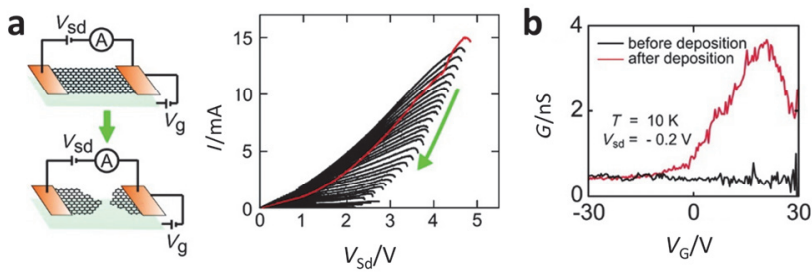

图 30 (a) 在少层石墨片中形成纳米尺寸的间隙(顶部)之前和之后(底 部)反馈控制的电动加热过程示意图, 以及电流电压 $(I-V)$ (绿色箭头)的 演变. 第一个 $I-V$ 曲线以红色显示. (b) 在 $10 \mathrm{~K}$ 下由 $9 \mathrm{Accm}$ 分子桥接 之前和之后的电导与所施加的纳米间隙电极的背栅极电压的关系曲 线 ${ }^{[113]}$

Figure 30 (a) Schematic of the feedback-controlled electroburning process, before (top) and after (bottom), the formation of nanometer sized gaps in few-layer graphite flakes. and Current-voltage $(I-V)$ traces of the evolution (green arrow) of the feedback-controlled electroburning. The first $I-V$ trace is displayed in red. (b) Conductance as a function of the applied back-gate voltage of the nanogapped electrodes bridged by 9 Accm molecules at $10 \mathrm{~K}$. While the empty nanogap electrodes show no dependence of the applied back-gate voltage, a clear conductance modulation as a function of $V_{\mathrm{g}}$ is observed after deposition of $9 \mathrm{Accm}$ molecules ${ }^{[113]}$

稳定性，且制备简单，有望代替常规的金属电极.

\section{4 单分子识别化学物种}

近年来, 对一些化学物种的识别逐渐成为人们研究

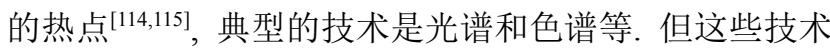
通常需要对大量分子进行测试统计, 所以单分子水平的 识别十分重要. Kawai 等 ${ }^{[114]}$ 利用 MCBJ 技术, 通过测量 单个氨基酸分子的电导识别了 20 余种多肽中的氨基酸, 如图 31 所示. 实验与理论十分相符.
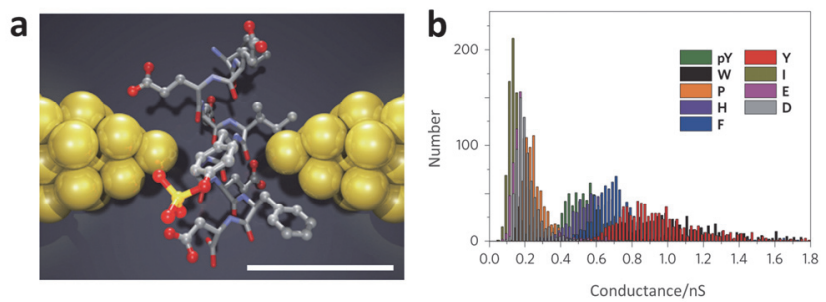

图 31 (a) MCBJ 技术制备分子结器件示意图. (b) 一定数量的不同氨 基酸的电导直方图 ${ }^{[114]}$

Figure 31 (a) Schematic diagram of preparation of molecular junction devices by MCBJ. (b) Conductance histogram of a certain number of different amino acids ${ }^{[114]}$

Guo 等 ${ }^{[15]}$ 利用石墨烯与羰基封端分子形成的单分 子结的电流变化监测其与羟氨溶液中的亲核加成反应. 随着反应的不断进行，由于中间态和初态的能级不同而 导致电流出现规律性的波动, 如图 32 所示. 通过观察实 时电流中的两个明显波动可以直接掌握单一分子级别 的基本化学反应的动态过程. 并且所制备的器件稳定, 能承受化学处理和外部刺激. 该方法可应用于化学与生 物化学领域.
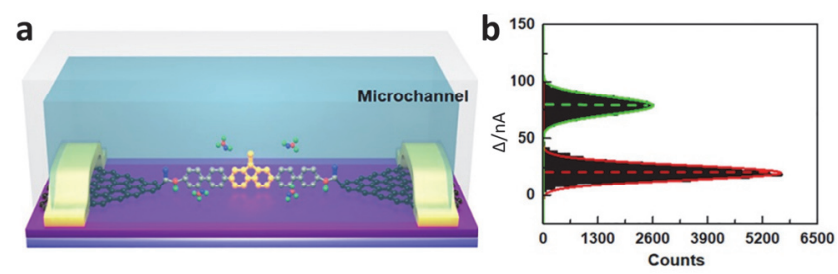

图 32 (a) 使用自制微通道进行单分子动力学表征的实时测量装置示 意图. (b) 显示双峰电流分布的电流值直方图 ${ }^{[15]}$

Figure 32 (a) Schematic diagram of real-time measurement setup with a home-made microchannel for single-molecule dynamics characterization. (b) Corresponding histogram of current values, showing a bimodal current distribution ${ }^{[115]}$

\section{5 商业化应用}

上面总结了分子电子学器件的几个尚处探索中的 潜在应用. 而分子电子器件的实际商业化应用已经实 现. McCreery 等 ${ }^{[16,1117]}$ 报道了基于碳膜电极和共价连接 的分子层的电子器件. 他们巧妙地把这种器件的相对平 坦的非线性伏安特性用于电吉他的限幅元件中. 分子器 件的伏安特性可以改变声音输出的奇偶谐波分布, 从而 改变电吉他的音色. 基于分子器件的限幅元件相比于传 统二极管的限幅元件在相同奇偶谐波分布时产生出比 
传统二极管限幅元件更柔和的音色, 如图 33 所示. 由于 分子结的电学行为很容易通过分子层厚度来改变, 因 此, 使用不同的分子结器件组成的限幅元件可以实现一 系列的音色调节. 这是迄今为止分子结器件唯一在实际 中的应用. 展现出了分子器件诱人的前景.
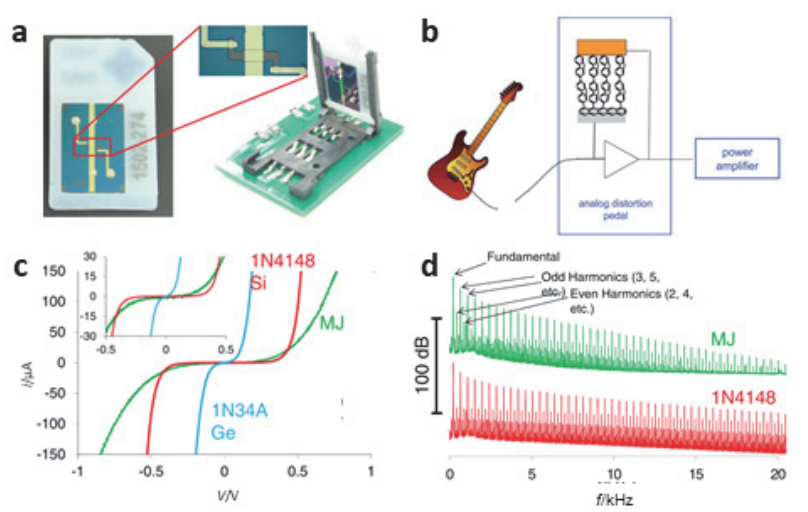

图 33 (a) 使用 SIM 卡引脚排列制作分子结的照片, 以简化测试并集 成到模拟电路中. (b) 将分子结器件应用于电吉他的放大器元件的示 意图. (c) 分子结器件与传统二极管器件的 $I-V$ 特性. (d) 不同频率下, 传统二极管与分子结器件的奇偶谐波分布 ${ }^{[116,117]}$

Figure 33 (a) Photograph of the molecular junctions fabricated using a SIM-card pin-out to simplify testing and integration into analog circuits, as shown at right. (b) Schematic diagram of applying a molecular junction device to an amplifier component of an electric guitar. (c) $I-V$ characteristics of molecular junction devices and conventional diode devices. (d) Odd and even harmonic distribution of conventional diode and molecular junction limiting components at different frequencies ${ }^{[116,117]}$

\section{5 总结与展望}

综上所述, 近十几年以来, 分子电子学的基础研究 及其应用已经取得了非常丰富的成果. 由于篇幅限制, 本文展示的有关分子电子学器件的制备、电荷传输机理 的表征以及器件的应用等方面的进展只能是管中窥豹, 是这一领域诸多进展中很小的一部分. 很多分子电子学 的激动人心的进展不得不舍弃, 例如, 分子结的热电功 能 ${ }^{[18]}$, 基于非弹性隧穿的非弹性谱 ${ }^{[119]}$ 和基于分子结中 电子涨落的散粒噪声谱等 ${ }^{[120]}$. 更完整的进展可以参考 Xiang 等 ${ }^{[24]}$ 的详细的综述和 Juan Cuevas 的分子电子学 专著 2017 年第二版 ${ }^{[25]}$.

最后, 我们想指出这一领域有待进一步探索和解决 的问题: 第一, 无论是单分子器件还是单分子层器件, 在制备和测试方面, 都存在器件稳定性相对较低且重复 性差, 对环境等外界因素影响较敏感等问题. 因此, 如 何提高器件的稳定性和重复性, 寻找合适的封装技术, 都是分子电子学走向实际应用在技术上迫切需要进一 步探索和解决的问题. 第二, 目前大部分的分子结器件 仍然主要关注电导的变化, 分子(作为隧穿势垒)所起的 作用较单一. 而实际上分子的本征特征从原理上来说可 以产生很多特殊的伏安特性, 从而为新型非传统器件提 供基础. 这些特殊伏安特性的分子器件仍有待进一步的 实验探索. 最后, 将分子电子学器件应用到实际中时,
如何解决分子电子学器件与主流硅基器件的兼容性问 题. 这些都是需要我们进一步探索和解决的问题.

\section{作者简介}

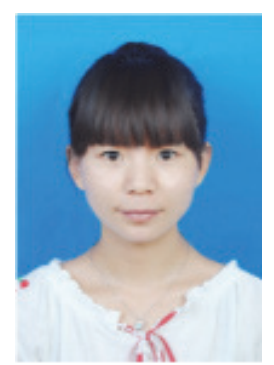

许晓娜, 在读硕士研究生. 主要从事分子电子学器件电 荷输运机理的研究.

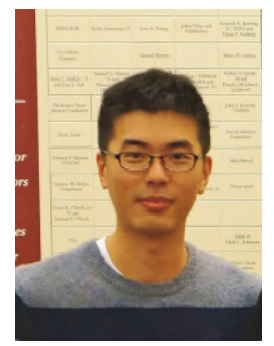

于曦, 天津大学理学院化学系, 天津市分子光电科学重点 实验室教授, 博导. 2012 年获麻省大学阿默斯特分校化学系博 士，之后去以色列 Weizmann 研究所材料与界面系，做博士后. 主要研究方向: 分子尺度电子学, 电荷传递-分子振动相互作 用, 量子输运与量子动力学, 有机电子学与柔性电子学, 自组 装光电功能材料.

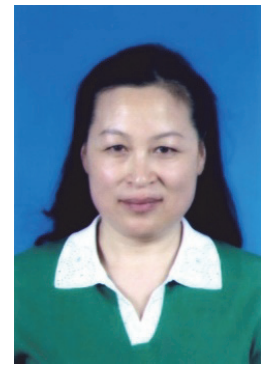

朱艳英, 燕山大学理学院物理系教授, 博导. 主要从事光 镊及表面增强拉曼方向的研究.

\section{References}

[1] Feynman, R. Engineering and Science 1960, 23, 8.

[2] Ratner, M. A.; Aviram, A. Chem. Phys. Lett. 1974, 29, 277.

[3] Jiang, L.; Huang, G. F.; Li, H. X.; Li, X. F.; Hu, W. P.; Liu, Y. Q.; Zhu, D. B. Prog. Chem. 2005, 17, 172 (in Chinese). (江浪, 黄桂芳, 李洪祥, 李小凡, 胡文平, 刘云圻, 朱道本, 化学进展, 2005, 17, 172.)

[4] Ai, Y.; Zhang, H. L. Acta Phys-Chim. Sin. 2012, 28, 2237. (in Chinese). (艾勇, 张浩力，物理化学学报，2012，28, 2237.)

[5] Zhou, C.; Reed, M. A.; Muller, C. J.; Burgin, T. P.; Tour, J. M. Science 1997, 278, 3.

[6] Yang, W. R.; Jones, M. W.; Li, X.; Eggers, P. K.; Tao, N. J.; Gooding, J.; Paddon-Row, M. N. J. Phys. Chem. C 2008, 112, 9072.

[7] Vilan, A.; Aswal, D.; Cahen, D. Chem. Rev. 2017, 117, 4248

[8] Chen, S.; Liu, Y.; Chen, J. Chem. Soc. Rev. 2014, 43, 5372. 
[9] Sun, L.; Diaz-Fernandez, Y. A.; Gschneidtner, T. A.; Westerlund, F.; Lara-Avila, S.; Moth-Poulsen, K. Chem. Soc. Rev. 2014, 43, 7378.

[10] Kuo, C. T.; Su, L. C.; Chen, C. H. J. Am. Chem. Soc. 2014, 61, 101.

[11] Ratner, M. Nature Nanotech. 2013, 8, 378.

[12] Zimbovskaya, N. A.; Pederson, M. R. Phys. Rep. 2011, 509, 1

[13] Aradhya, S. V.; Venkataraman, L. Nature Nanotech. 2013, 8, 399.

[14] Claridge, S. A.; Schwartz, J. J.; Weiss, P. S. ACS Nano 2011, 5, 693.

[15] Song, H.; Reed, M. A.; Lee, T. Adv. Mater. 2011, 23, 1583.

[16] Chen, F.; Tao, N. J. Acc. Chem. Res. 2009, 42, 429.

[17] Heath, J. R. Annu. Rev. Mater. Res. 2009, 39, 1.

[18] Poulsen, K. M.; Bjornholm, T. Nature Nanotech. 2009, 4, 551

[19] McCreery, R. L.; Bergren, A. J. Adv. Mater. 2009, 21, 4303.

[20] Akkerman, H. B.; de Boer, B. J. Phys. Condens. Matter. 2008, 20, 013001 .

[21] Nitzan, A.; Ratner, M. A. Science 2003, 300, 1384.

[22] Su, T. A.; Neupane, M.; Steigerwald, M. L.; Venkataraman, L.; Nuckolls, C. Nat. Rev. Mater. 2016, 1, 16002.

[23] Zhang, X.; Li, T. Chin. Chem. Lett. 2017, 28, 2058.

[24] Xiang, D.; Wang, X.; Jia, C.; Lee, T.; Guo, X. Chem. Rev. 2016, 116, 4318.

[25] Cuevas, J. C.; Scheer, E. Molecular Electronics, 2nd ed., USA: World Scientific Publishing Co, 2017, pp. $1 \sim 826$.

[26] Li, J. C.; Wu, J. Z.; Zhou, C.; Gong, X. Acta Phys.-Chim. Sin. 2013, 29,1123 (in Chinese). (李建昌, 吴隽稚, 周成, 宫兴, 物理化学学 报, 2013, 29, 1123).

[27] Yang, Y.; Liu, J. Y.; Yan, R. W.; Wu, D. Y.; Tian, Z. Q. Chem. J. Chin. Univ. 2015, 36, 9 (in Chinese). (杨扬, 刘俊扬, 晏润文, 田中 群, 高等学校化学学报, 2015, 36, 9).

[28] Chen, F.; Hihath, J.; Huang, Z.; Li, X.; Tao, N. J. Annu. Rev. Phys. Chem. 2007, 58, 535.

[29] Xu, B. Q.; Tao, N. J. Science 2003, 301, 1221.

[30] Guo, S.; Hihath, J.; Diez-Perez, I.; Tao, N. J. J. Am. Chem. Soc. 2011, 133, 19189.

[31] Baldea, I. Phys. Rev. B 2012, 85, 9222.

[32] Chen, F.; Li, X.; Hihath, J.; Huang, Z.; Tao, N. J. J. Am. Chem. Soc. 2006, $128,15874$.

[33] Hines, T.; Diez-Perez, I.; Nakamura, H.; Shimazaki, T.; Asai, Y.; Tao, N. J. J. Am. Chem. Soc. 2013, 135, 3319.

[34] Li, H.; Su, T. A.; Zhang, V.; Steigerwald, M. L.; Nuckolls, C.; Venkataraman, L. J. Am. Chem. Soc. 2015, 137, 5028.

[35] Dell, E. J.; Capozzi, B.; DuBay, K. H.; Berkelbach, T. C.; Moreno, J. R.; Reichman, D. R.; Venkataraman, L.; Campos, L. M. J. Am. Chem. Soc. 2013, 135, 11724.

[36] Wold, D. J.; Frisbie, C. D. J. Am. Chem. Soc. 2000, 122, 2970.

[37] Aradhya, S. V.; Frei, M.; Hybertsen, M. S.; Venkataraman, L. Nat. Mater. 2012, 11,872 .

[38] Nazin, G. V.; Wu, S. W.; Ho, W. PNAS 2005, 102, 8832.

[39] Zhou, J.; Chen, F.; Xu, B. Q. J. Am. Chem. Soc. 2009, 131, 10439.

[40] Zhou, J.; Chen, G.; Xu, B. Q. J. Phys. Chem. C 2010, 114, 8587.

[41] Moreland, J.; Ekin, J. W. J. Appl. Phys. 1985, 58, 3888.

[42] Muller, C. J.; van Ruitenbeek, J. M.; de Jongh, L. J. Phys. Rev. Lett. 1992, 69, 140

[43] Tian, J. H.; Liu, B.; Li, X.; Yang, Z. L.; Ren, B.; Wu, S. T.; Tao, N. J.; Tian, Z. Q. J. Am. Chem. Soc. 2006, 128, 14748.

[44] Xiang, D.; Jeong, H.; Kim, D.; Lee, T.; Cheng, Y.; Wang, Q.; Mayer, D. Nano Lett. 2013, 13, 2809.

[45] Holmlin, R. E.; Ismagilov, R. F.; Haag, R.; Mujica, V.; Ratner, M. A.; Rampi, M. A.; Whitesides, G. M. Angew. Chem., Int. Ed. Engl. 2001, 40, 2316.

[46] Thuo, M. M.; Reus, W. F.; Nijhuis, C. A.; Barber, J. R.; Kim, C.; Schulz, M. D.; Whitesides, G. M. J. Am. Chem. Soc. 2011, 133, 2962.

[47] Nijhuis, C. A.; Reus, W. F.; Barber, J. R.; Dickey, M. D.; Whitesides, G. M. Nano Lett. 2010, 10, 3611 .

[48] Chiechi, R. C.; Weiss, E. A.; Dickey, M. D.; Whitesides, G. M. Angew. Chem., Int. Ed. Engl. 2008, 47, 142.

[49] Senthil kumar, K.; Jiang, L.; Nijhuis, C. A. RSC Adv. 2017, 7, 14544.

[50] Walker, A. V.; Tighe, T. B.; Haynie, B. C.; Uppili, S.; Winograd, N.; Allara, D. L. J. Phys. Chem. B 2005, 109, 11263.

[51] Mahmoud, A. M.; Bergren, A. J.; Pekas, N.; McCreery, R. L. Adv. Funct. Mater, 2011, 21, 2273.

[52] Zhu, Z.; Daniel, T. A.; Maitani, M.; Cabarcos, O. M.; Allara, D. L.; Winograd, N. J. Am. Chem. Soc. 2006, 128, 13710.

[53] Walker, A. V.; Tighe, T. B.; Cabarcos, O. M.; Reinard, M. D.;
Haynie, B. C.; Uppili, S.; Winograd, N.; Allara, D. L. J. Am. Chem. Soc. 2004, 126, 3954.

[54] DeIonno, E.; Tseng, H. R.; Harvey, D. D.; Stoddart, J. F.; Heath, J. R. J. Phys. Chem. B 2006, 110, 7609.

[55] Bonifas, A. P.; McCreery, R. L. Nat. Nanotechnol. 2010, 5, 612.

[56] Honciuc, A.; Metzger, R. M.; Gong, A.; Spangler, C. W. J. Am. Chem. Soc. 2007, 129, 8310 .

[57] Bonifas, A. P.; McCreery, R. L. Nano Lett. 2011, 11, 4725.

[58] Akkerman, H. B.; Blom, P. W. M.; de Leeuw, D. M.; de Boer, B. Nature 2006, 441, 69.

[59] Katsouras, I.; Piliego, C.; Blom, P. W. M.; Leeuwa, D. M. Nanoscale 2013, 5, 9882.

[60] Puebla-Hellmann, G.; Venkatesan, K.; Mayor, M.; Lörtscher, E. Nature 2018, 559, 232.

[61] Noy, G.; Ophir, A.; Selzer, Y. Angew. Chem., Int. Ed. Engl. 2010, 49, 5734.

[62] Rigaut, S. Dalton Trans. 2013, 42, 15859.

[63] Choi, S. H.; Kim, B.; Frisbie, C. D. Science 2008, 320, 1482.

[64] Wang, W.; Lee, T.; Reed, M. A. Phys. Rev. B 2003, 68, 035416-1.

[65] Jeremy, B. K.; Beebe, M.; Frisbie, C. D.; Kushmerick, J. G. ACS Nano 2008, 2, 827.

[66] Vilan, A.; Cahen, D.; Kraisler, E. ACS Nano 2013, 7, 695.

[67] Huisman, E. H.; Guedon, C. M.; Wees, B. J.; van der Molen, S. J. Nano Lett. 2009, 9, 3909.

[68] Jia, C. C.; Guo, X. Chem. Soc. Rev. 2013, 42, 5642.

[69] Baldea, I.; Xie, Z.; Frisbie, C. D. Nanoscale 2015, 7, 10465.

[70] Widawsky, J. R.; Kamenetska, M.; Klare, J.; Nuckolls, C.; Steigerwald, M. L.; Hybertsen, M. S.; Venkataraman, L. Nanotechnology 2009, 20, 434009 .

[71] Lee, W.; Reddy, P. Nanotechnology 2011, 22, 485703.

[72] Baldea, I. Nanoscale. 2013, 5, 9222.

[73] Chen, J.; Calvet, L. C.; Reed, M. A.; Carr, D. W.; Grubisha, D. S.; Bennett, D. W. Chem. Phys. Lett. 1999, 313, 741.

[74] Selzer, Y.; Cabassi, M. A.; Mayer, T. S.; Allara, D. L. J. Am. Chem. Soc. 2004, 126, 4052.

[75] Choi, S. H.; Risko, C.; Delgado, M. C. R.; Kim, B.; Bredas, J. L.; Frisbie, C.D. J. Am. Chem. Soc. 2010, 132, 4358.

[76] Hill, M. G.; Treadway, C. R.; Barton, J. K. Chem. Phys. 2002, 281, 409 .

[77] Kelley, S. O.; Barton, J. K. Science 1999, 283, 375.

[78] Eley, D. D.; Spivey, D. I. Trans. Faraday Soc. 1962, 58, 411

[79] Genereux, J. C.; Barton, J. K. Chem. Rev. 2010, 110, 1642.

[80] Xiang, L.; Palma, J. L.; Bruot, C.; Mujica, V.; Ratner, M. A.; Tao, N. J. Nat. Chem. 2015, 7, 221.

[81] Bostick, C. D.; Mukhopadhyay, S.; Pecht, I.; Sheves, M.; Cahen, D.; Lederman, D. Rep. Prog. Phys. 2018, 81, 026601.

[82] Amdursky, N.; Marchak, D.; Sepunaru, L.; Pecht, I.; Sheves, M.; Cahen, D. Adv. Mater. 2014, 26, 7142.

[83] Andrews. D. Q.; Solomon, G. C.; Goldsmith, R. H.; Hansen, T.; Wasielewski, M. R.; Duyne, R. P.; Ratner, M. A. J. Am. Chem. Soc. 2008, 130, 17301 .

[84] Hong, W. J.; Valkenier, H.; Meszaros, G.; Manrique, D. Z.; Mishchenko, A.; Putz, A.; Garcia, P. M.; Lambert, C. J.; Hummelen, J. C.; Wandlowski, T. Beilstein J. Nanotechnol. 2011, 2, 699.

[85] Fracasso, D.; Valkenier, H.; Hummelen, J. C.; Solomon, G. C.; Chiechi, R. C. J. Am. Chem. Soc. 2011, 133, 9556

[86] Guedon, C. M.; Valkenier, H.; Markussen, T.; Thygesen, K. S.; Hummelen, J. C.; Molen, S. J. Nat. Nanotechnol. 2012, 7, $304 .$.

[87] Rabache, V.; Chaste, J.; Petit, P.; Della Rocca, M. L.; Martin, P.; Lacroix, J. C.; McCreery, R. L.; Lafarge, P. J. Am. Chem. Soc. 2013, $135,10218$.

[88] Manrique, D. Z.; Huang, C.; Baghernejad, M.; Zhao, X.; AlOwaedi, O. A.; Sadeghi, H.; Kaliginedi, V.; Hong, W. J.; Wandlowski, M.; Gulcur, T.; Bryce, M. R.; Lambert, C. J. Nat. Commun. 2015, 6, 6389

[89] Liu, X. S.; Sangtarash, S.; Reber, D.; Zhang, D.; Sadeghi, H.; Shi, J.; Xiao, Z.; Hong, W. J.; Lambert, C. J.; Liu, S. X. Angew. Chem., Int. Ed. Engl. 2017, 56, 173.

[90] Zhang, P.; Chen, L. C.; Zhang, Z. Q.; Cao, J. J.; Tang, C.; Liu, J.; Duan, L. L.; Huo, Y.; Shao, X.; Hong, W. J.; Zhang, H. L. J. Am. Chem. Soc. 2018, 140, 6531.

[91] Bai, J.; Daaoub, A.; Sangtarash, S.; Li, X.; Tang, Y.; Zou, Q.; Sadeghi, H.; Liu, S.; Huang, X.; Tan, Z.; Liu, J.; Yang, Y.; Shi, J.; Meszaros, G.; Chen, W.; Lambert, C.; Hong, W. J. Nat. Mater. 2019.

[92] Liu, J.; Huang, X.; Wang, F.; Hong, W. J. Acc. Chem. Res. 2019, 52, 
151.

[93] Dong, H.; Deng, N.; Chen, P. Y. World Science and Technology Research and Development 2005, 27, 1 (in Chinese). (董浩, 邓宁, 陈 培毅, 世界科技研究与发展, 2005, 27, 1).

[94] Dhirani, A.; Lin, P. H.; Sionnest, P. G.; Zehner, R. W.; Sita, L. R. J. Chem. Phys. 1997, 106, 6.

[95] Metzger, R. M.; Xu, T.; Peterson, I. R. J. Phys. Chem. B 2001, 105, 7280 .

[96] Diez-Perez, I.; Hihath, J.; Lee, Y.; Yu, L.; Adamska, L.; Kozhushner, M. A.; Oleynik, I. I.; Tao, N. J. Nat. Chem. 2009, 1, 635.

[97] Kushmerick, J. G.; Whitaker, C. M.; Pollack, S. K.; T Schull,. L.; Shashidhar, R. Nat. Nanotechnol. 2004, 15, S489.

[98] Wang, K.; Zhou, J.; Hamill, J. M.; Xu, B. Q. J. Chem. Phys. 2014, $141,054712$.

[99] Batra, A.; Darancet, P.; Chen, Q.; Meisner, J. S.; Widawsky, J. R.; Neaton, J. B.; Nuckolls, C.; Venkataraman, L. Nano Lett. 2013, 13, 6233.

[100] Nijhuis, C. A.; Reus, W. F.; Whitesides, G. M. J. Am. Chem. Soc. 2010, 132, 18386.

[101] Nijhuis, C. A.; Reus, W. F.; Siegel, A. C.; Whitesides, G. M. J. Am. Chem. Soc. 2011, 133, 15397.

[102] Chen, X.; Roemer, M.; Yuan, L.; Du, W.; Thompson, D.; Del Barco, E.; Nijhuis, C. A. Nat. Nanotechnol. 2017, 12, 797.

[103] Katsonis, N.; Kudernac, T.; Walko, M.; Molen, S. J.; Wees, B. J.; Feringa, B. L. Adv. Mater. 2006, 18, 1397.

[104] Ikeda, M.; Tanifuji, N.; Yamaguchi, H.; Irie, M.; Matsuda, K. Chem. Commun. 2007, 1355.

[105] Whalley, A. C.; Steigerwald, M. L.; Guo, X. F.; Nuckolls, C. J. Am. Chem. Soc. 2007, 129, 12590.

[106] Jia, C.; Wang, J.; Yao, C.; Cao, Y.; Zhong, Y.; Liu, Z.; Liu, Z.; Guo, X. F. Angew. Chem., Int. Ed. Engl. 2013, 52, 8666.
[107] Migliore, A.; Jia, C. C.; Xin, N.; Huang, S. Y.; Wang, J. Y.; Yang, Q.; Wang, S. P.; Chen, H. L.; Wang, D. M.; Feng, B. Y.; Liu, Z. R.; Zhang, G. Y.; Qu, D. H.; Tian, H.; Ratner, M. A.; Xu, H. Q.; Nitzan, A.; Guo, X. F. Science 2016, 352, 1443.

[108] Javey, A.; Guo, J.; Wang, Q.; Lundstrom, M.; Dai, H. Nature 2003, 424,654 .

[109] Cui, Y.; Lieber, C. M. Science 2001, 291, 851.

[110] Damle, P.; Rakshit, T.; Paulsson, M.; Datta, S. IEEE T. Nanotechnol. 2002, 1,145 .

[111] Xu, B. Q.; Xiao, X. Y.; Yang, X. M.; Zang, L.; Tao, N. J. J. Am. Chem. Soc. 2005, 127, 2386.

[112] Song, H.; Kim, Y.; Jang, Y. H.; Jeong, H.; Reed, M. A.; Lee, T. Nature 2009, 462, 1039.

[113] Prins, F.; Barreiro, A.; Ruitenberg, J. W.; Seldenthuis, J. S.; Aliaga-Alcalde, N.; Vandersypen, L. M.; van der Zant, H. S. Nano Lett. 2011, 11, 4607.

[114] Ohshiro, T.; Tsutsui, M.; Yokota, K.; Furuhashi, M.; Taniguchi, M.; Kawai, T. Nat. Nanotechnol. 2014, 9, 835.

[115] Guan, J.; Jia, C.; Li, Y.; Liu, Z.; Wang, J.; Yang, Z.; Gu, C.; Su, D.; Houk, K. N.; Zhang, D.; Guo, X. F. Sci. Adv. 2018, 4, 2177.

[116] Bergren, A. J.; Zeer-Wanklyn, L.; Semple, M.; Pekas, N.; Szeto, B.; McCreery, R. L. J. Phys. Condens. Matter. 2016, 28, 094011.

[117] McCreery, R. L.; Bergren, A.; Morteza-Najarian, A.; Sayed, S. Y.; Yan, H. Faraday Discuss 2014, 172, 9.

[118] Rincon-Garcia, L.; Evangeli, C.; Rubio-Bollinger, G.; Agrait, N. Chem. Soc. Rev. 2016, 45, 4285.

[119] Kim, Y.; Song, H. Appl. Spectrosc. Rev. 2016, 51, 603.

[120] Xiang, D.; Sydoruk, V.; Vitusevich, S.; Petrychuk, M. V.; Offenhaeusser, A.; Kochelap, V. A.; Belyaev, A. E.; Mayer, D. Appl. Phys. Lett. 2015, 106, 063702-1. 